\title{
Analytic Expressions and Bounds for Special Functions and Applications in Communication Theory
}

\author{
Paschalis C. Sofotasios, Member, IEEE, Theodoros A. Tsiftsis, Senior Member, IEEE, Yury A. Brychkov, \\ Steven Freear, Senior Member, IEEE, Mikko Valkama, Member, IEEE, \\ and George K. Karagiannidis, Fellow, IEEE
}

\begin{abstract}
This paper is devoted to the derivation of novel analytic expressions and bounds for a family of special functions that are useful in wireless communication theory. These functions are the well-known Nuttall $Q$-function, incomplete Toronto function, Rice $I e$-function, and incomplete Lipschitz-Hankel integrals. Capitalizing on the offered results, useful identities are additionally derived between the above functions and Humbert, $\Phi_{1}$, function as well as for specific cases of the Kampé de Fériet function. These functions can be considered as useful mathematical tools that can be employed in applications relating to the analytic performance evaluation of modern wireless communication systems, such as cognitive radio, cooperative, and free-space optical communications as well as radar, diversity, and multiantenna systems. As an example, new closed-form expressions are derived for the outage probability over nonlinear generalized fading channels, namely, $\alpha-\eta-\mu$, $\alpha-\lambda-\mu$, and $\alpha-\kappa-\mu$ as well as for specific cases of the $\eta-\mu$ and $\lambda-\mu$ fading channels. Furthermore, simple expressions are
\end{abstract}

Manuscript received March 19, 2014; revised September 8, 2014; accepted September 9, 2014. Date of publication September 26, 2014; date of current version November 18, 2014. This work was supported in part by the Academy of Finland through the Project entitled Digitally-Enhanced RF for Cognitive Radio Devices under Grant 251138, in part by the Russian Foundation for Fundamental Research under Project 13-07-00334, and in part by the U.K Engineering and Physical Sciences Research Council. This paper was presented at the 2010 IEEE ICWITS, 2011 IEEE SBMO/IMOC, 2011 ATNAC, 2011 IEEE INDICON, and 2013 ATC.

P. C. Sofotasios was with the School of Electronic and Electrical Engineering, University of Leeds, Leeds LS2 9JT, U.K. He is now with the Department of Electronics and Communications Engineering, Tampere University of Technology, Tampere 33720, Finland, and also with the Department of Electrical and Computer Engineering, Aristotle University of Thessaloniki, Thessaloniki 54124, Greece (e-mail: p.sofotasios@ieee.org).

T. A. Tsiftsis is with the Department of Electrical Engineering, Technological Educational Institute of Central Greece, Lamia 35100, Greece (e-mail: tsiftsis@teilam.gr)

Y. A. Brychkov is with the Dorodnicyn Computing Center, Russian Academy of Sciences, Moscow 119991, Russia (e-mail: brychkov@ccas.ru).

$\mathrm{S}$. Freear is with the School of Electronic and Electrical Engineering, University of Leeds, Leeds LS2 9JT, U.K. (e-mail: s.freear@leeds.ac.uk).

M. Valkama is with the Department of Electronics and Communications Engineering, Tampere University of Technology, Tampere 33720, Finland (e-mail: mikko.e.valkama@tut.fi)

G. K. Karagiannidis is with the Department of Electrical and Computer Engineering, Khalifa University, Abu Dhabi 127788, United Arab Emirates, and also with the Department of Electrical and Computer Engineering, Aristotle University of Thessaloniki, Thessaloniki 54124, Greece (e-mail: geokarag@ieee.org).

Communicated by A. Lozano, Associate Editor for Communications.

Color versions of one or more of the figures in this paper are available online at http://ieeexplore.iee.org.

Digital Object Identifier 10.1109/TIT.2014.2360388 presented for the channel capacity for the truncated channel inversion with fixed rate and corresponding optimum cutoff signal-to-noise ratio for single-antenna and multiantenna communication systems over Rician fading channels. The accuracy and validity of the derived expressions is justified through extensive comparisons with respective numerical results.

Index Terms-Special functions, wireless communication theory, fading channels, emerging wireless technologies, multiantenna systems, outage probability, truncated channel inversion.

\section{INTRODUCTION}

$\mathbf{I}$ T IS widely known that special functions constitute invaluable mathematical tools in most fields of natural sciences and engineering. In the broad area of wireless communications, their utilization often allows the derivation of useful expressions for important performance measures such as error probability, channel capacity and higher-order statistics (HOS). The computational realization of such expressions is typically straightforward since the majority of special functions, that are used in digital communications, are included as built-in functions in popular mathematical software packages such as MAPLE, MATLAB and MATHEMATICA. Among others, the Marcum $Q$-function, $Q_{m}(a, b)$, the Nuttall $Q$-function, $Q_{m, n}(a, b)$, the Rice $I e$-function, $I e(k, x)$, the incomplete Toronto function (ITF), $T_{B}(m, n, r)$, and the incomplete Lipschitz-Hankel integrals (ILHIs), $Z e_{m, n}(x ; a)$, were proposed several decades ago [1]-[22] and have been largely involved in communication theory and in the analytic performance evaluation of wireless communications systems [23]-[37] and the references therein.

More specifically, the $Q_{m}(a, b)$ function was proposed by Marcum in [1] and [2] and became widely known in digital communications by applications relating to wireless transmission over fading or non-fading media [5]-[11], [23]. Its basic properties and identities were reported in [9] and several upper and lower bounds were proposed in [10] and [38]-[52]. Furthermore, semi-analytic representations and approximations were given in [53]-[56] while various properties were investigated in [42], [43], [46], [50], [57], and [58]. Exact analytic expressions for the special cases that $m$ is a nonnegative integer and half-integer were derived in [7], [46], and [58], respectively, whereas general expressions in terms of the confluent Appell function were derived in [57] and 
also in [59] in the context of deriving closed-form expressions for the bivariate Nakagami- $m$ distribution and the distribution of the minimum eigenvalue of correlated non-central Wishart matrices.

In the same context, the $Q_{m, n}(a, b)$ function was firstly proposed in [9] and constitutes a generalization of the Marcum $Q$-function. It is defined by a semi-infinite integral representation and it can be expressed in terms of the $Q_{m}(a, b)$ function and the modified Bessel function of the first kind, $I_{n}(x)$, for the special case that the sum of its indices is a real odd positive integer i.e. $(m+n+1) / 2 \in \mathbb{N}$. Establishment of further properties, monotonicity criteria and the derivation of lower and upper bounds along with a closed-form expression for the case that $m \pm 0.5 \in \mathbb{N}$ and $n \pm 0.5 \in \mathbb{N}$ were reported in [45], [46], [60], and [61]. Likewise, the incomplete Toronto function is a special function, which was proposed by Hatley in [12]. It constitutes a generalization of the Toronto function, $T(m, n, r)$, and includes the $Q_{m}(a, b)$ function as a special case. Its definition is given by a finite integral while alternative representations include two infinite series that were proposed in [13]. The incomplete Toronto function has also been useful in wireless communications as it has been employed in applications relating to statistical analysis, radar systems, signal detection and estimation as well as in error probability analysis [3], [4], [14].

The Rice $I e$-function is also a special function of similar analytic representation to the Marcum and Nuttall $Q$-functions. It was firstly proposed by S. O. Rice in [15] and has been applied in investigations relating to zero crossings, angle modulation systems, radar pulse detection and error rate analysis of differentially encoded systems [16]-[18], [62]. It is typically defined by a finite integral while alternative representations include two infinite series which involve the modified Struve function and an expression in terms of the Marcum $Q$-function [17], [18], [62], [63]. Finally, the $Z e_{m, n}(x ; a)$ integrals constitute a general class of incomplete cylindrical functions that have been encountered in analytic solutions of numerous problems in electromagnetic theory [20], [22]-and the references therein. Their general representation is given in a non-infinite integral form and it accounts accordingly for the Bessel function of the first kind, $J_{n}(x)$, the Bessel function of the second kind, $Y_{n}(x)$, and their modified counterparts, $I_{n}(x)$ and $K_{n}(x)$, respectively. In the context of wireless communication systems, the ILHIs have been utilized in the OP over generalized multipath fading channels as well as in the error rate analysis of MIMO systems under imperfect channel state information (CSI) employing adaptive modulation, transmit beamforming and maximal ratio combining (MRC), [29], [33], [64].

Nevertheless, in spite of the undoubted importance of the $Q_{m, n}(a, b), T_{B}(m, n r), I e(k, x)$ functions and $Z e_{m, n}(x ; a)$ integrals, they are all neither available in tabulated form nor are included as built-in functions in widely used mathematical software packages. As a consequence, their utilization becomes rather intractable and laborious to handle both algebraically and computationally. Motivated by this, analytic results on these special functions and integrals were reported in [65]-[74]. In the same context, the present work is devoted to elaborating substantially on these results aiming to derive a comprehensive mathematical framework that consists of numerous analytic expressions and bounds for the above special functions and integrals. The offered results have a versatile algebraic representation and can be useful in applications relating to natural sciences and engineering, including conventional and emerging wireless communications.

In more details, the contributions of the present paper are listed below:

- Closed-form expressions and simple polynomial approximations are derived for the $Q_{m, n}(a, b), T_{B}(m, n, r)$, $I e(k, x)$ functions and the $I e_{m, n}(x ; a)$ integrals. ${ }^{1}$ These expressions are valid for all values of the involved parameters and can readily reduce to exact infinite series representations.

- Closed-form upper bounds are derived for the respective truncation errors of the proposed polynomial and series representations.

- Simple closed-form expressions are derived for specific cases of the $T_{B}(m, n, r)$ function and the $I e_{m, n}(x ; a)$ integrals.

- Capitalizing on the derived expressions, generic closedform upper and lower bounds are derived for the $T_{B}(m, n, r)$ function and the $I e_{m, n}(x ; a)$ integrals.

- Simple closed-form upper and lower bounds are proposed for the $I e(k, x)$ function which under certain range of values become accurate approximations.

- Simple closed-form upper bounds are proposed for the $Q_{m, n}(a, b), T_{B}(m, n, r)$ functions and $I e_{m, n}(x ; a)$ integrals which for certain range of values can serve as particularly tight approximations.

- Novel closed-form identities are deduced relating specific cases of the Kampé de Fériet (KdF) and Humbert, $\Phi_{1}$, functions with the above special functions. These identities are useful because although $\Phi_{1}$ and particularly $\mathrm{KdF}$ functions are rather generic functions that are capable of representing numerous other special functions, yet, they are currently neither explicitly tabulated nor built-in functions in popular mathematical software packages such as MATLAB, MAPLE and MATHEMATICA.

- The offered results are applied in the context of digital communications for deducing respective analytic expressions for: $i$ ) the outage probability (OP) over non-linear generalized fading, namely, $\alpha-\eta-\mu, \alpha-\lambda-\mu$ and $\alpha-\kappa-\mu$ fading channels; ii) the OP for $\eta-\mu$ and $\lambda-\mu$ fading channels for the special case that the value of $\mu$ is integer or half-integer; $i$ ii) the channel capacity for the truncated channel inversion with fixed rate (TIFR) adaptive transmission technique of single-and multiantenna systems over Rician fading channels; iv) the optimum cut-off SNR for the aforementioned TIFR scenario in the case of single-input single-output (SISO), multiple-input single-output (MISO) and single-input multiple-output (SIMO) systems.

\footnotetext{
${ }^{1}$ This work considers only the $I e_{m, n}(x ; a)$ case i.e. the $I_{n}(x)$ functionbased $Z e_{m, n}(x ; a)$ integrals. However, the offered analytic expressions can be readily extended for the case of $J e_{m, n}(x ; a), Y e_{m, n}(x ; a)$ and $K e_{m, n}(x ; a)$ with the aid of the standard identities of the Bessel functions.
} 
To the best of the Authors' knowledge, the offered results have not been previously reported in the open technical literature. The remainder of this paper is organized as follows: New expressions are derived for the Nuttall $Q$-function in Sec. II. Sec. III and Sec. IV are devoted to the derivation of closed-form expressions and bounds for the ITF and Rice $I e-$ function, respectively. Analytic results for the ILHIs are derived in Section $\mathrm{V}$ while simple identities for special cases of the KdF and Humbert $\Phi_{1}$ functions are proposed in Sec. VI. Finally, applications in the context of wireless communications along with the necessary discussions are provided in Section VII while closing remarks are given in Section VIII.

\section{New Closed-Form Expressions AND Bounds FOR THE NUTTALL $Q$-FUNCTION}

Definition 1: For $m, n, a, b \in \mathbb{R}^{+}$, the Nuttall $Q$-function is defined by the following semi-infinite integral representation [9, eq. (86)],

$$
Q_{m, n}(a, b) \triangleq \int_{b}^{\infty} x^{m} e^{-\frac{x^{2}+a^{2}}{2}} I_{n}(a x) \mathrm{d} x .
$$

The Nuttall $Q$-function constitutes a generalization of the well-known Marcum $Q$-function. The normalized Nuttall $Q-$ function is expressed as,

$$
\mathcal{Q}_{m, n}(a, b)=\frac{Q_{m, n}(a, b)}{a^{n}}
$$

which for the special case that $m=1$ and $n=0$, reduces to the Marcum $Q$-function, namely,

$$
Q_{1}(a, b) \triangleq \int_{b}^{\infty} x e^{-\frac{x^{2}+a^{2}}{2}} I_{0}(a x) \mathrm{d} x
$$

and thus, $Q_{1,0}(a, b)=\mathcal{Q}_{1,0}(a, b)=Q_{1}(a, b)=Q(a, b)$. In addition, for the special case that $n=m-1$ it follows that,

$$
\begin{aligned}
\mathcal{Q}_{m, m-1}(a, b) & =Q_{m}(a, b) \\
& =\frac{1}{a^{m-1}} \int_{b}^{\infty} x^{m} e^{-\frac{x^{2}+a^{2}}{2}} I_{m-1}(a x) \mathrm{d} x
\end{aligned}
$$

and thus, $Q_{m, m-1}(a, b)=a^{m-1} Q_{m}(a, b)$. Likewise, when $m$ and $n$ are positive integers, the following recursion formula is valid [11, eq. (3)],

$$
\begin{aligned}
Q_{m, n}(a, b)= & a Q_{m-1, n+1}(a, b)+b^{m-1} e^{-\frac{a^{2}+b^{2}}{2}} I_{n}(a b) \\
& +(m+n-1) Q_{m-2, n}(a, b)
\end{aligned}
$$

along with the finite series representation in [11, eq. (8)]. Nevertheless, the validity of this series is not general because it is restricted to the special case that the sum of $m$ and $n$ is an odd positive integer i.e. $m+n \in \mathbb{N}$.

\section{A. A Closed-Form Expression in Terms} of the Kampé de Fériet Function

Theorem 1: For $m, n, a \in \mathbb{R}$ and $b \in \mathbb{R}^{+}$, the Nuttall $Q-$ function can be expressed as follows,

$$
\begin{aligned}
Q_{m, n}(a, b)= & \frac{a^{n} \Gamma\left(\frac{m+n+1}{2}\right){ }_{1} F_{1}\left(\frac{m+n+1}{2}, 1+n, \frac{a^{2}}{2}\right)}{n ! e^{\frac{a^{2}}{2}} 2^{\frac{n-m+1}{2}}} \\
& -\frac{a^{n} b^{m+n+1} F_{1,1}^{1,0}\left(\begin{array}{l}
\frac{m+n+1}{2}:-,-: \\
\frac{m+n+3}{2}: n+1,-:
\end{array} \frac{a^{2} b^{2}}{4},-\frac{b^{2}}{2}\right)}{n !(m+n+1) 2^{n} e^{\frac{a^{2}}{2}}}
\end{aligned}
$$

where $\Gamma(a),{ }_{1} F_{1}(a, b, x)$ and $F_{., .}(\because ., .$,$) denote the (complete)$ Gamma function, the Kummer confluent hypergeometric function and the KdF function, respectively [75]-[82].

Proof: The proof is provided in Appendix A.

\section{B. A Simple Polynomial Representation}

In spite of the general usefulness of (7), its presence in integrands along with other elementary and/or special functions can lead to intractable integrals due to the absence of relatively simple representations and properties for the $F_{., .} \cdot(\because ., .$,$) function. Therefore, it is evident that a simple$ approximative formula that is valid for all values of the involved parameters is additionally useful.

Proposition 1: For $m, n, a \in \mathbb{R}$ and $b \in \mathbb{R}^{+}$, the $Q_{m, n}(a, b)$ function can be accurately approximated as follows,

$$
Q_{m, n}(a, b) \simeq \sum_{l=0}^{p} \frac{a^{n+2 l} \Gamma(p+l) p^{1-2 l} \Gamma\left(\frac{m+n+2 l+1}{2}, \frac{b^{2}}{2}\right)}{l !(n+l) ! 2^{\frac{n-m+2 l+1}{2}}(p-l) ! e^{\frac{a^{2}}{2}}}
$$

which for the special case that $(m+n+1) / 2 \in \mathbb{N}$, it can be equivalently expressed as,

$$
Q_{m, n}(a, b) \simeq \sum_{l=0}^{p} \sum_{k=0}^{L} \frac{\mathcal{A} a^{n+2 l} b^{2 k} \Gamma(p+l) p^{1-2 l} \Gamma(L+l+1)}{l ! k ! \Gamma(n+l+1)(p-l) ! 2^{l+k} e^{-\frac{a^{2}+b^{2}}{2}}}
$$

where

$$
L=\frac{m+n-1}{2}+l
$$

and

$$
\mathcal{A}=a^{n} 2^{\frac{m-n-1}{2}} e^{-\frac{a^{2}+b^{2}}{2}}
$$

with $p$ denoting the corresponding truncation term, and $\Gamma(a, x)$ is the upper incomplete Gamma function [63].

Proof: The proof is provided in Appendix B.

Remark 1: The coefficients of the series in [83, eq. (19)] differ from the series in [63, eq. (8.445)] by the terms $p^{1-2 l} \Gamma(p+l) /(p-l)$ !. Therefore, as $p \rightarrow \infty$, these terms vanish and as a result (8) and (9) reduce to the following exact infinite series representations,

$$
Q_{m, n}(a, b)=\sum_{l=0}^{\infty} \frac{a^{n+2 l} e^{-\frac{a^{2}}{2}} \Gamma\left(\frac{m+n+2 l+1}{2}, \frac{b^{2}}{2}\right)}{l ! \Gamma(n+l+1) 2^{\frac{n-m+2 l+1}{2}}}
$$


and

$$
Q_{m, n}(a, b)=\sum_{l=0}^{\infty} \sum_{k=0}^{\frac{m+n-1}{2}+l} \frac{a^{n+2 l} b^{2 k} 2^{\frac{m-n-1}{2}} \Gamma\left(\frac{m+n+1}{2}+l\right)}{l ! k ! \Gamma(n+l+1) 2^{l+k} e^{\frac{a^{2}+b^{2}}{2}}}
$$

respectively to (8) and (9).

Remark 2: By setting $n=m-1$ in (8) and recalling that $\mathcal{Q}_{m, n}(a, b)=Q_{m, n}(a, b) / a^{n}$ and $\mathcal{Q}_{m, m-1}(a, b)=Q_{m}(a, b)$, a new simple approximation is deduced for the Marcum $Q$-function,

$$
Q_{m}(a, b) \simeq \sum_{l=0}^{p} \frac{a^{2 l} \Gamma(p+l) p^{1-2 l} \Gamma\left(m+l, \frac{b^{2}}{2}\right)}{l ! \Gamma(m+l) 2^{l}(p-l) ! e^{\frac{a^{2}}{2}}}
$$

which for $m \in \mathbb{N}$ it can reduce to,

$$
Q_{m}(a, b) \simeq \sum_{l=0}^{p} \sum_{k=0}^{m+l-1} \frac{\Gamma(p+l) p^{1-2 l} a^{2 l} b^{2 k}}{l ! k !(p-l) ! 2^{l+k} e^{\frac{a^{2}+b^{2}}{2}}} .
$$

Based on Remark 1, as $p \rightarrow \infty$, equations (16) and (17) become exact infinite series, namely,

$$
Q_{m}(a, b)=\sum_{l=0}^{\infty} \frac{a^{2 l} \Gamma\left(m+l, \frac{b^{2}}{2}\right)}{l ! \Gamma(m+l) 2^{l} e^{\frac{a^{2}}{2}}}
$$

and

$$
Q_{m}(a, b)=\sum_{l=0}^{\infty} \sum_{k=0}^{m+l-1} \frac{a^{2 l} b^{2 k}}{l ! k ! 2^{l+k}} e^{-\frac{a^{2}+b^{2}}{2}}
$$

respectively.

\section{Truncation Error}

The proposed expressions converge rather quickly and their accuracy is proportional to the value of $p$. However, determining the involved truncation error analytically is particularly advantageous for ensuring certain accuracy levels when applied in analyses related to wireless communications.

Lemma 1: For $m, n, a \in \mathbb{R}$ and $b \in \mathbb{R}^{+}$, the following inequality can serve as a closed-form upper bound for the truncation error of the $Q_{m, n}(a, b)$ function in (8),

$$
\begin{aligned}
\epsilon_{t} \leq & \sum_{k=0}^{\lceil n\rceil_{0.5}-1} \frac{(-1)^{\lceil n\rceil_{0.5}} \Gamma\left(2\lceil n\rceil_{0.5}-k-1\right) \mathcal{I}_{\lceil m\rceil_{0.5},\lceil n\rceil_{0.5}}^{k}(a, b)}{k ! \Gamma\left(\lceil n\rceil_{0.5}-k\right)(2 a)^{-k} \sqrt{\pi} 2^{\lceil n\rceil_{0.5}-\frac{1}{2}} a^{2\lceil n\rceil_{0.5}-1}} \\
& -\sum_{l=0}^{p} \frac{p a^{n+2 l} \Gamma(p+l) \Gamma\left(\frac{m+n+2 l+1}{2}, \frac{b^{2}}{2}\right)}{l ! p^{2 l}(n+l) ! 2^{\frac{n-m+2 l-1}{2}}(p-l) ! e^{\frac{a^{2}}{2}}}
\end{aligned}
$$

where,

$$
\begin{aligned}
\mathcal{I}_{m, n}^{k}(a, b)= & \sum_{l=0}^{m-n+k}\left(\begin{array}{c}
m-n+k \\
l
\end{array}\right) \frac{(-1)^{k} 2^{\frac{l-1}{2}}}{a^{l-m-k}} \\
\times & \left\{(-1)^{m-n-l-1} \Gamma\left(\frac{l+1}{2}, \frac{(b+a)^{2}}{2}\right)\right. \\
& -[\operatorname{sgn}(b-a)]^{l+1} \gamma\left(\frac{l+1}{2}, \frac{(b-a)^{2}}{2}\right) \\
& \left.+\Gamma\left(\frac{l+1}{2}\right)\right\}
\end{aligned}
$$

where $\gamma(a, x)$ is the lower incomplete Gamma function and

$$
\lceil x\rceil_{0.5} \triangleq\lceil x-0.5\rceil+0.5
$$

with $\lceil$.$\rceil denoting the integer ceiling function.$

Proof: The truncation error of (8) is expressed by definition as follows:

$$
\begin{aligned}
\epsilon_{t}= & \sum_{l=p+1}^{\infty} \frac{a^{n+2 l} \Gamma(p+l) p^{1-2 l} \Gamma\left(\frac{m+n+2 l+1}{2}, \frac{b^{2}}{2}\right)}{l !(n+l) ! 2^{\frac{n-m+2 l+1}{2}}(p-l) ! e^{\frac{a^{2}}{2}}} \\
= & \underbrace{\sum_{l=0}^{\infty} \frac{a^{n+2 l} \Gamma(p+l) p^{1-2 l} \Gamma\left(\frac{m+n+2 l+1}{2}, \frac{b^{2}}{2}\right)}{l ! e^{\frac{a^{2}}{2}}(n+l) ! 2^{\frac{n-m+2 l+1}{2}}(p-l) !}}_{\mathcal{I}_{1}} \\
& -\sum_{l=0}^{p} \frac{a^{n+2 l} \Gamma(p+l) p^{1-2 l} \Gamma\left(\frac{m+n+2 l+1}{2}, \frac{b^{2}}{2}\right)}{l !(n+l) ! e^{\frac{a^{2}}{2}} 2^{\frac{n-m+2 l+1}{2}}(p-l) !} .
\end{aligned}
$$

Given that the $\mathcal{I}_{1}$ series is infinite and based on the proposed series in [83], the terms

$$
\frac{\Gamma(p+l) p^{1-2 l}}{\Gamma(p-l+1)}
$$

vanish which yields,

$$
\begin{aligned}
\mathcal{I}_{1} & =\sum_{l=0}^{\infty} \frac{a^{n+2 l} e^{-\frac{a^{2}}{2}} \Gamma\left(\frac{m+n+2 l+1}{2}, \frac{b^{2}}{2}\right)}{l ! \Gamma(n+l+1) 2^{\frac{n-m+2 l+1}{2}}} \\
& =Q_{m, n}(a, b) .
\end{aligned}
$$

It is recalled here that according to [46, eq. (19)],

$$
Q_{m, n}(a, b) \leq Q_{\lceil m\rceil_{0.5},\lceil n\rceil_{0.5}}(a, b)
$$

Therefore, by substituting (25) in (23) and then in (21) one obtains the following inequality,

$$
\begin{aligned}
\epsilon_{t} \leq & Q_{\lceil m\rceil_{0.5},\lceil n\rceil_{0.5}}(a, b) \\
& -\sum_{l=0}^{p} \frac{a^{n+2 l} e^{-\frac{a^{2}}{2}} \Gamma(p+l) p^{1-2 l} \Gamma\left(\frac{m+n+2 l+1}{2}, \frac{b^{2}}{2}\right)}{l ! \Gamma(n+l+1) 2^{\frac{n-m+2 l+1}{2}} \Gamma(p-l+1)} .
\end{aligned}
$$

The upper bound for the $Q_{m, n}(a, b)$ function can be expressed in closed-form with the aid of [46, Corollary 1]. Therefore, by substituting in (26) yields (28), which completes the proof. $\square$

Remark 3: For the specific case that $n=m-1$ and given that $\mathcal{Q}_{m, m-1}(a, b)=Q_{m}(a, b)$, the following upper bound is obtained for the truncation error of the Marcum $Q$-function representations in (16) and (17),

$$
\begin{aligned}
\epsilon_{t} \leq & \sum_{l=1}^{m-\frac{1}{2}} \sum_{k=0}^{l-1} \frac{(-1)^{l} b^{k}(l-k)_{l-1}\left[1-(-1)^{k} e^{2 a b}\right]}{k ! \sqrt{\pi} 2^{l-2 k-\frac{1}{2}} a^{2 l-k-1} e^{\frac{(a+b)^{2}}{2}}}+Q(b+a) \\
& +Q(b-a)-\sum_{l=0}^{p} \frac{a^{2 l} \Gamma(p+l) p^{1-2 l} \Gamma\left(m+l, \frac{b^{2}}{2}\right)}{l ! \Gamma(m+l) 2^{l}(p-l) ! e^{\frac{a^{2}}{2}}}
\end{aligned}
$$

where $Q(x)$ denotes the one dimensional Gaussian $Q$-function [75]. By following the same methodology 
as in Lemma 1, a respective upper bound can be also deduced for the truncation error of the infinite series in Remark 1, namely,

$$
\begin{aligned}
& \epsilon_{t} \leq \sum_{k=0}^{\lceil n\rceil_{0.5}-1} \frac{(-1)^{\lceil n\rceil_{0.5}} \Gamma\left(2\lceil n\rceil_{0.5}-k-1\right) \mathcal{I}_{\lceil m\rceil_{0.5},\lceil n\rceil_{0.5}}^{k}(a, b)}{k ! \Gamma\left(\lceil n\rceil_{0.5}-k\right)(2 a)^{-k} \sqrt{\pi} 2^{\lceil n\rceil_{0.5}-\frac{1}{2}} a^{2\lceil n\rceil_{0.5}-1}} \\
& -\sum_{l=0}^{p} \frac{a^{n+2 l} \Gamma\left(\frac{m+n+2 l+1}{2}, \frac{b^{2}}{2}\right)}{l !(n+l) ! 2^{\frac{n-m+2 l-1}{2}} e^{\frac{a^{2}}{2}}}
\end{aligned}
$$

\section{A Tight Upper Bound and Approximation}

Proposition 2: For $a, b, m, n \in \mathbb{R}^{+}$and for the special cases that either $b \rightarrow 0$ or $a, m, n \geq \frac{3}{2} b$, the following closedform upper bound for the Nuttall $Q$-function is valid,

$$
Q_{m, n}(a, b) \leq \frac{a^{n} \Gamma\left(\frac{m+n+1}{2}\right)}{n ! 2^{\frac{n-m+1}{2}} e^{\frac{a^{2}}{2}}}{ }_{1} F_{1}\left(\frac{m+n+1}{2}, n+1, \frac{a^{2}}{2}\right)
$$

which becomes an accurate approximation when $a, m$, $n \geq \frac{5}{2} b$.

Proof: Given that (8) becomes an exact infinite series as $p \rightarrow \infty$ and with the aid of the monotonicity property $\Gamma(a, x) \leq \Gamma(x), a \in \mathbb{R}^{+}$, the $Q_{m, n}(a, b)$ can be upper bounded as follows,

$$
Q_{m, n}(a, b) \leq \underbrace{\sum_{l=0}^{\infty} \frac{a^{n+2 l} e^{-\frac{a^{2}}{2}} \Gamma\left(\frac{m+n+2 l+1}{2}\right)}{l ! \Gamma(n+l+1) 2^{\frac{n-m+2 l+1}{2}}}}_{\mathcal{I}_{2}} .
$$

By expressing each Gamma function as,

$$
\Gamma(x+l)=(x)_{l} \Gamma(x)
$$

and carrying out some algebraic manipulations one obtains,

$$
\mathcal{I}_{2}=\frac{\Gamma\left(\frac{m+n+1}{2}\right) e^{\frac{-a^{2}}{2}}}{n ! 2^{\frac{n-m+1}{2}}} \sum_{l=0}^{\infty} \frac{\left(\frac{m+n+1}{2}\right)_{l} a^{n+2 l}}{l !(n+1)_{l} 2^{l}} .
$$

The above infinite series can be expressed in terms of Kummer's hypergeometric function in [63, eq. (9.14.1)]. Therefore, by performing the necessary change of variables and substituting (32) into (30) yields (29), which completes the proof.

It is noted here that similar expressions to the $Q_{m, n}(a, b)$ function can be also deduced for the $\mathcal{Q}_{m, n}(a, b)$ function by applying the identity

$$
Q_{m, n}(a, b)=a^{n} \mathcal{Q}_{m, n}(a, b)
$$

which corresponds to dividing equations (8), (9), (28), and (29) by $a^{n}$.

The behaviour of the offered results is depicted in Table I along with respective results from numerical integrations for comparisons. The polynomial series was truncated after 20 terms and one can notice the excellent agreement between analytical and numerical results. This is also verified by the value of the corresponding absolute relative error,

$$
\epsilon_{r} \triangleq \frac{\left|Q_{m, n}(a, b)-\tilde{Q}_{m, n}(a, b)\right|}{Q_{m, n}(a, b)}
$$

TABLE I

ACCURACY OF PROPOSED EXPRESSIONS For THE $Q_{m, n}(a, b)$ FUnCTION

\begin{tabular}{|c|c|c|c|c|}
\hline FUNCTION & EXACT & Eq. (7) & Eqs. (8), (9) & Eqs. (29) \\
\hline \hline$Q_{0.7,0.3}(0.6,0.4)$ & 0.6956 & 0.6956 & 0.6956 & 0.7458 \\
\hline$Q_{1.6,1.4}(0.6,0.4)$ & 0.2890 & 0.2890 & 0.2890 & 0.2898 \\
\hline$Q_{1.2,1.8}(0.6,0.4)$ & 0.1295 & 0.1295 & 0.1295 & 0.1299 \\
\hline$Q_{0.7,0.3}(0.9,0.4)$ & 0.7580 & 0.7580 & 0.7580 & 0.8035 \\
\hline$Q_{1.6,1.4}(0.6,1.3)$ & 0.2360 & 0.2360 & 0.2360 & 0.2898 \\
\hline$Q_{1.2,1.8}(2.0,2.0)$ & 0.5380 & 0.5380 & 0.5380 & 0.7403 \\
\hline
\end{tabular}

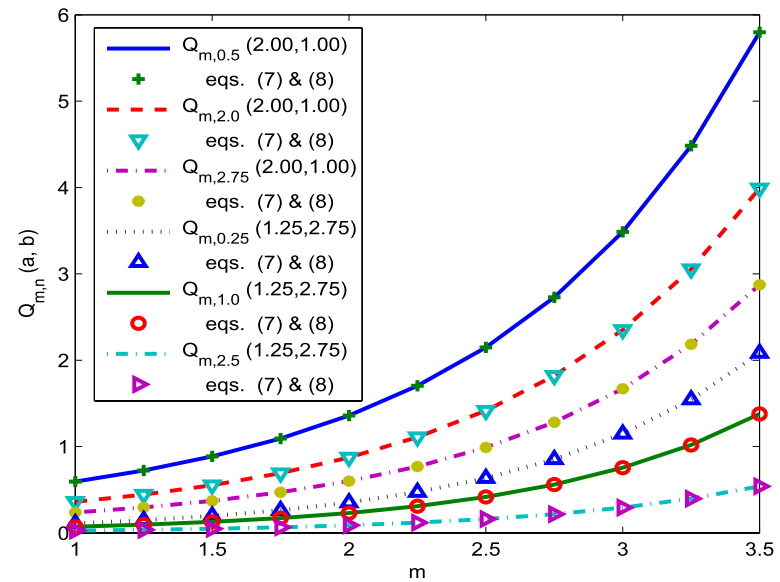

(a)

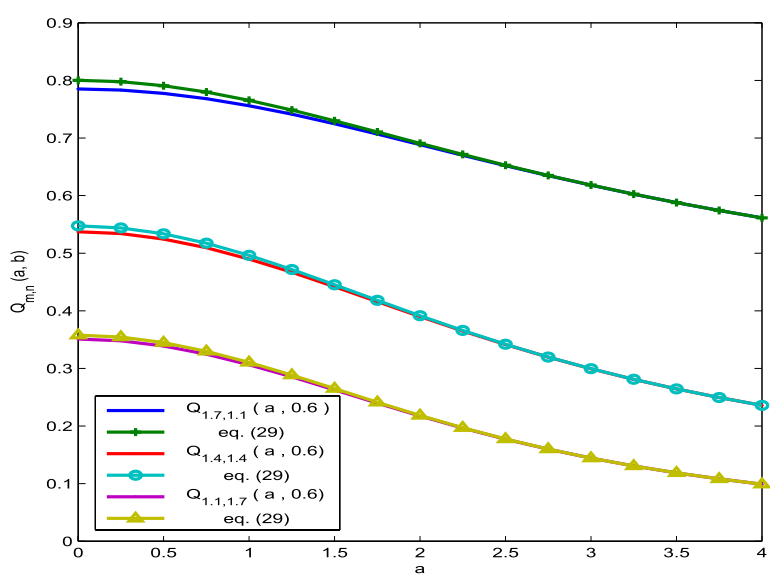

(b)

Fig. 1. Behaviour and accuracy of the normalized Nuttall $Q$-function in the proposed equations (7), (8) \& (29). (a) $\mathcal{Q}_{m, n}(a, b)$ in (7) and (8). (b) $\mathcal{Q}_{m, n}(a, b)$ in (29).

which is typically smaller than $\epsilon_{r}<10^{-11}$. It is also shown that (29) appears to be rather accurate particularly for high values of $a$.

The behavior of (7) and (8) is also illustrated in Fig. 1a for arbitrary values of the involved parameters whereas Fig. 1b depicts the accuracy of (29). It is clearly observed that (29) upper bounds the $\mathcal{Q}_{m, n}(a, b)$ tightly and becomes a rather accurate approximation as $a$ increases asymptotically. Moreover, both (8) and (29) are tighter than the closed-form bounds proposed in [46], since they are in adequate match with the respective theoretical results for most cases. 


\section{New Closed-Form EXPRessions fOR THE INCOMPLETE TORONTO FUNCTION}

Definition 2: For $m, n, r, B \in \mathbb{R}^{+}$, the incomplete Toronto function is defined as follows,

$$
\begin{aligned}
& T_{B}(m, n, r) \triangleq 2 r^{n-m+1} e^{-r^{2}} \int_{0}^{B} t^{m-n} e^{-t^{2}} I_{n}(2 r t) \mathrm{d} t . \\
& \text { he ITF has been also a useful special function in wir. }
\end{aligned}
$$

The ITF has been also a useful special function in wireless communications. When $B \rightarrow \infty$, it reduces to the (complete) Toronto function,

$$
T(m, n, r) \triangleq 2 r^{n-m+1} e^{-r^{2}} \int_{0}^{\infty} t^{m-n} e^{-t^{2}} I_{n}(2 r t) \mathrm{d} t
$$

while for the specific case that $n=(m-1) / 2$ it is expressed in terms of the Marcum $Q$-function namely,

$$
T_{B}\left(m, \frac{m-1}{2}, r\right)=1-Q_{\frac{m+1}{2}}(r \sqrt{2}, B \sqrt{2}) .
$$

Alternative representations include two infinite series in [13]; however, to the best of the Authors' knowledge no study has been reported in the open technical literature for the convergence and truncation of these series.

\section{A. Special Cases}

Theorem 2: For $r \in \mathbb{R}, B \in \mathbb{R}^{+}, m \in \mathbb{N}, n \pm 0.5 \in \mathbb{N}$ and $m>n$, the following closed-form expression is valid for the incomplete Toronto function,

$$
\begin{aligned}
T_{B}(m, n, r)= & \sum_{k=0}^{n-\frac{1}{2}} \sum_{l=0}^{L} \frac{\Gamma\left(n+k+\frac{1}{2}\right)(L-k) ! 2^{-2 k} r^{-2 k-l)}}{2 \sqrt{\pi} k ! l ! \Gamma\left(n-k+\frac{1}{2}\right)(L-k-l) !} \\
& \times\left\{(-1)^{m-l} \gamma\left[\frac{l+1}{2},(B+r)^{2}\right]\right. \\
& \left.+(-1)^{k} \gamma\left[\frac{l+1}{2},(B-r)^{2}\right]\right\}
\end{aligned}
$$

where $L=m-n-\frac{1}{2}$.

Proof: The proof is provided in Appendix C.

In the same context, a similar closed-form expression can be derived for the case that $m-2 n$ is an odd positive integer. To this end, it is firstly essential to algebraically link the incomplete Toronto function with the Nuttall $Q$-function, which is provided in the following Lemma.

Lemma 2: For $m, n, r \in \mathbb{R}$ and $B \in \mathbb{R}^{+}$, the $T_{B}(m, n, r)$ function can be algebraically related to the $Q_{m, n}(a, b)$ function by the following representation,

$$
T_{B}(m, n, r)=\frac{\Gamma\left(\frac{m+1}{2}\right){ }_{1} F_{1}\left(n+\frac{1-m}{2}, n+1,-r^{2}\right)}{n ! r^{m-2 n-1}}
$$

Proof: The proof is provided in Appendix D.

Lemma 2 is subsequently employed in the proof of the following theorem.

Theorem 3: For $r \in \mathbb{R}, B \in \mathbb{R}^{+}, m \in \mathbb{Z}^{+}, n \in \mathbb{N}, m>2 n$ and $\frac{m}{2}-n \notin \mathbb{N}$, the following closed-form expression is valid for the incomplete Toronto function,

$$
\begin{aligned}
T_{B}(m, n, r)= & \frac{\Gamma\left(\frac{m+1}{2}\right)}{r^{m-2 n-1} n !}{ }_{1} F_{1}\left(n+\frac{1-m}{2}, n+1,-r^{2}\right) \\
& -\sum_{l=1}^{\frac{m-1}{2}-n} \sum_{j=0}^{\frac{m-1}{2}-n-l} \frac{r^{n+l} 2^{2 l+2 j} \Gamma\left(\frac{m+1}{2}\right)}{\Gamma(l) r^{m}\left(\frac{m-1}{2}-j-n\right)} 1-l \\
& \times \frac{B^{n+l+2 j+1} I_{n+l-1}(2 r B)}{\Gamma(n+l+j+1)} \\
& -\sum_{l=1}^{\frac{m+1}{2}-n} \frac{r^{2 n+2 l-m-1} \Gamma\left(\frac{m+1}{2}-n\right)}{\Gamma(l) \Gamma(n+l)\left(\frac{m+1}{2}\right)_{1-n-l}} \\
& \times\left\{Q_{1}(\sqrt{2} r, \sqrt{2} B)+\sum_{i=1}^{n+l-1} \frac{b^{i} I_{i}(2 r B)}{r^{i} e^{r^{2}+B^{2}}}\right\}
\end{aligned}
$$

where $Q_{1}(a, b)=Q(a, b)$ denotes the Marcum $Q$-function of the first order.

Proof: By utilizing (39) and [11, eq. (8)] and after basic algebraic manipulations it follows that,

$$
\begin{aligned}
T_{B}(m, n, r)= & \frac{\Gamma\left(\frac{m+1}{2}\right){ }_{1} F_{1}\left(n+\frac{1-m}{2}, n+1,-r^{2}\right)}{n ! r^{m-2 n-1}} \\
& -\sum_{l=1}^{\frac{m+1}{2}-n} \frac{r^{2(n+l)} \Gamma\left(\frac{m+1}{2}-n\right) Q_{n+l}(\sqrt{2} r, \sqrt{2} B)}{r^{m+1} 2^{\frac{1-l}{2}} \Gamma(l) \Gamma(n+l)\left(\frac{m+1}{2}\right)_{1-n-l}} \\
& -\sum_{l=1}^{\frac{m-1}{2}-n} \sum_{j=0}^{\frac{m-1}{2}-n-l} \frac{\Gamma\left(\frac{m-1}{2}-j-n\right) b^{n+l+2 j+1}}{r^{m-n-l} \Gamma(l) \Gamma(1-n-l-j)} \\
& \times \Gamma\left(\frac{m+1}{2}\right) e^{-\frac{r^{2}+B^{2}}{2}} I_{n+l-1}(2 r B) .
\end{aligned}
$$

Given that $n \in \mathbb{N}$, the $Q_{m}(a, b)$ function can be equivalently expressed in terms of the $Q_{1}(a, b)$ function according to [11, eq. (12)]. To this effect, by performing the necessary variable transformation and substituting in (41) yields (40), which completes the proof.

\section{B. Closed-Form Bounds}

Lemma 3: For $m, n, B \in \mathbb{R}^{+}, r \in \mathbb{R}$ and $m \geq n$, the following inequalities can serve as upper and lower bounds to the incomplete Toronto function,

$$
T_{B}(m, n, r) \leq T_{B}\left(\lceil m\rceil,\lfloor n\rfloor_{0.5}, r\right)
$$

and

$$
T_{B}(m, n, r) \geq T_{B}\left(\lfloor m\rfloor,\lceil n\rceil_{0.5}, r\right)
$$

where $\lfloor$.$\rfloor denotes the integer floor function.$

Proof: Based on the monotonicity properties of the Toronto function, $T_{B}(m, n, r)$ is strictly increasing w.r.t $m$ and strictly decreasing w.r.t $n$. Furthermore, two half-integer rounding operators were given in [46, eq. (18)], namely,

$$
\lfloor n\rfloor_{0.5}=\lfloor n+0.5\rfloor-0.5
$$

and

$$
\lceil n\rceil_{0.5}=\lceil n-0.5\rceil+0.5 \text {. }
$$


By also recalling that (38) is valid for $m \in \mathbb{N}$ and $n \pm 0.5 \in \mathbb{N}$, it follows that $T_{B}\left(\lceil m\rceil,\lfloor n\rfloor_{0.5}, r\right)$ and $T_{B}\left(\lfloor m\rfloor,\lceil n\rceil_{0.5}, r\right)$ can be expressed in closed-form for any value of $m, n, r, B$ and can hence serve as a closed-form bounds for $T_{B}(m, n, r)$. Thus, by applying the above floor and ceiling functions in (38), equations (42) and (43) are obtained, which completes the proof.

\section{A Closed-Form Expression in Terms of the Kampé de Fériet Function}

A more generalized closed-form expression for the ITF, that does not impose any restrictions to the involved parameters, can be derived in terms of the KdF function.

Theorem 4: For $m, n, r \in \mathbb{R}, B \in \mathbb{R}^{+}$and $m+n>-1$, the incomplete Toronto function can be expressed as follows,

$$
\begin{aligned}
T_{B}(m, n, r)= & \frac{2 r^{2 n-m+1} B^{m+1}}{n !(m+1)} e^{r^{2}} \\
& \times F_{1,1}^{1,0}\left(\begin{array}{l}
\frac{m+1}{2}:-,-: \\
\frac{m+3}{2}: n+1,-:
\end{array} r^{2} B^{2},-B^{2}\right) .
\end{aligned}
$$

Proof: The proof is provided in Appendix E.

\section{A Simple Polynomial Representation}

The proposed specific and generalized expressions are rather useful in applications relating to wireless communications. However, a relatively simple and general representation for the ITF is additionally necessary for cases that the parameters of the $T_{B}(m, n, r)$ are required to be unrestricted and the algebraic representation of the function must be rather simple.

Proposition 3: For $m, n, r \in \mathbb{R}$ and $B \in \mathbb{R}^{+}$, the following polynomial approximation holds for the $T_{B}(m, n, r)$ function,

$$
T_{B}(m, n, r) \simeq \sum_{k=0}^{p} \frac{\Gamma(p+k) r^{2(n+k)-m+1} \gamma\left(\frac{m+1}{2}+k, B^{2}\right)}{k ! p^{2 k-1} \Gamma(p-k+1) \Gamma(n+k+1) e^{r^{2}}} .
$$

Proof: The proof follows from Theorem 2 and Proposition 1 and with the aid of [83, eq. (19)] and [63, eq. (8.350.1)].

Remark 4: By recalling that [83, eq. (19)] reduces to [63, eq. (8.445)] when $p \rightarrow \infty$, it immediately follows that (47) becomes an infinite series representation as $p \rightarrow \infty$, namely,

$$
T_{B}(m, n, r)=\sum_{k=0}^{\infty} \frac{r^{2(n+k)-m+1}}{k !(n+k) ! e^{r^{2}}} \gamma\left(\frac{m+1}{2}+k, B^{2}\right)
$$

which is exact.

\section{E. A Closed-Form Upper Bound for the Truncation Error}

A tight upper bound for the truncation error of (47) can be derived in closed-form.

Lemma 4: For $m, n, r \in \mathbb{R}, B \in \mathbb{R}^{+}$and $m>n$ the following closed-form inequality can serve as an upper bound for the truncation error in (47),

$$
\begin{aligned}
\epsilon_{t} \leq & \sum_{k=0}^{\lfloor n\rfloor_{0.5}^{-\frac{1}{2}}} \sum_{l=0}^{L} \frac{r^{-(2 k+l)}\left(\lfloor n\rfloor_{0.5}+k-\frac{1}{2}\right) !(L-k) !}{k ! l !\left(\lfloor n\rfloor_{0.5}-k-\frac{1}{2}\right) !(L-k-l) !} \\
& \times\left\{\frac{\gamma\left[\frac{l+1}{2},(B+r)^{2}\right]}{(-1)^{\lceil m\rceil-l} 2^{2 k+1}}+\frac{\gamma\left[\frac{l+1}{2},(B-r)^{2}\right]}{(-1)^{k} 2^{2 k+1}}\right\} \\
& -\sum_{k=0}^{p} \frac{\Gamma(p+k) r^{2(n+k)-m+1} \gamma\left(\frac{m+1}{2}+k, B^{2}\right)}{k ! p^{2 k-1} \Gamma(p-k+1) \Gamma(n+k+1) e^{r^{2}}} .
\end{aligned}
$$

Proof: Since the corresponding truncation error is expressed as

$$
\begin{aligned}
\epsilon_{t} & \triangleq \sum_{p+1}^{\infty} f(x) \\
& =\sum_{l=0}^{\infty} f(x)-\sum_{l=0}^{p} f(x)
\end{aligned}
$$

and given that (47) reduces to an exact infinite series as $p \rightarrow \infty$, it follows that,

$$
\begin{aligned}
\epsilon_{t}= & \underbrace{\sum_{k=0}^{\infty} \frac{r^{2(n+k)-m+1} \gamma\left(\frac{m+1}{2}+k, B^{2}\right)}{k ! \Gamma(n+k+1) e^{r^{2}}}}_{\mathcal{I}_{7}} \\
& -\sum_{k=0}^{p} \frac{\Gamma(p+k) r^{2(n+k)-m+1} \gamma\left(\frac{m+1}{2}+k, B^{2}\right)}{k ! p^{2 k-1} \Gamma(p-k+1) \Gamma(n+k+1) e^{r^{2}}} .
\end{aligned}
$$

It is noted that,

$$
\mathcal{I}_{7}=T_{B}(m, n, r) .
$$

To this effect and with the aid of (42), the $\epsilon_{t}$ can be upper bounded as follows:

$$
\begin{aligned}
\epsilon_{t} \leq & T_{B}\left(\lceil m\rceil,\lfloor n\rfloor_{0.5}, r\right) \\
& -\sum_{k=0}^{p} \frac{\Gamma(p+k) r^{2(n+k)-m+1} \gamma\left(\frac{m+1}{2}+k, B^{2}\right)}{k ! p^{2 k-1} \Gamma(p-k+1) \Gamma(n+k+1) e^{r^{2}}} .
\end{aligned}
$$

It is recalled here that the $T_{B}\left(\lceil m\rceil,\lfloor n\rfloor_{0.5}, r\right)$ function can be expressed in closed-form according to (38). Therefore, by substituting in (54) one obtains (55), which completes the proof.

Remark 5: By omitting the coefficients

$$
\frac{\Gamma(p+k) p^{1-2 k}}{\Gamma(p-k+1)}
$$

in the second term of (54) as $p \rightarrow \infty$, a closed-form upper bound can be deduced for the truncation error of the infinite series in (48), namely,

$$
\begin{aligned}
\epsilon_{t} \leq & \sum_{k=0}^{\lfloor n\rfloor_{0.5}^{-\frac{1}{2}}} \sum_{l=0}^{L} \frac{r^{-(2 k+l)}\left(\lfloor n\rfloor_{0.5}+k-\frac{1}{2}\right) !(L-k) !}{k ! l !\left(\lfloor n\rfloor_{0.5}-k-\frac{1}{2}\right) !(L-k-l) !} \\
& \times\left\{\frac{\gamma\left[\frac{l+1}{2},(B+r)^{2}\right]}{(-1)^{\lceil m\rceil-l} 2^{2 k+1}}+\frac{\gamma\left[\frac{l+1}{2},(B-r)^{2}\right]}{(-1)^{k} 2^{2 k+1}}\right\} \\
& -\sum_{k=0}^{p} \frac{r^{2(n+k)-m+1} \gamma\left(\frac{m+1}{2}+k, B^{2}\right)}{k ! \Gamma(n+k+1) e^{r^{2}}} .
\end{aligned}
$$


TABLE II

ACCURACY OF PROPOSED EXPRESSIONS FOR $T_{B}(m, n, r)$

\begin{tabular}{|c|c|c|c|c|}
\hline FUNCTION & EXACT & Eqs. (38), (3), (46) & Eq. (47) & Eq. (56) \\
\hline \hline$T_{3}(2.0,0.5,2.0)$ & 0.8695 & $0.8695, \mathrm{n} / \mathrm{a}, 0.8695$ & 0.8695 & 1.000 \\
\hline$T_{3}(3.0,1.5,2.0)$ & 0.7554 & $0.7554, \mathrm{n} / \mathrm{a}, 0.7554$ & 0.7554 & 0.8761 \\
\hline$T_{5}(2.0,0.5,2.0)$ & 0.9999 & $0.9999, \mathrm{n} / \mathrm{a}, 0.9999$ & 0.9999 & 1.0000 \\
\hline$T_{5}(3.0,1.5,2.0)$ & 0.8760 & $0.8760, \mathrm{n} / \mathrm{a}, 0.8760$ & 0.8760 & 0.8761 \\
\hline$T_{4}(3.0,1.0,2.0)$ & 0.9930 & n/a, $0.9930,0.9930$ & 0.9930 & 1.000 \\
\hline$T_{4}(5.0,2.0,2.0)$ & 0.9865 & n/a, $0.9865,0.9865$ & 0.9865 & 1.000 \\
\hline
\end{tabular}

\section{F. A Tight Closed-Form Upper Bound and Approximation}

Capitalizing on the algebraic representation of the $T_{B}(m, n, r)$ function, a simple closed-form upper bound is proposed which in certain cases becomes an accurate approximation.

Proposition 4: For $m, n, r \in \mathbb{R}, B \in \mathbb{R}^{+}$and $m, n, r \leq \frac{B}{2}$, the following inequality holds,

$$
T_{B}(m, n, r) \leq \frac{\Gamma\left(\frac{m+1}{2}\right){ }_{1} F_{1}\left(\frac{m+1}{2}, n+1, r^{2}\right)}{r^{m-2 n-1} \Gamma(n+1) e^{r^{2}}}
$$

which when $m, n, r \leq 2 B$, it can serve as a tight closed-form approximation.

Proof: The proof follows from Proposition 2 and with the aid of the monotonicity identity $\gamma(a) \geq \gamma(a, x)$.

The accuracy of the offered expressions is demonstrated in Table II (top of the page). The exact formulas are in full agreement with the respective numerical results which is also the case for the proposed polynomial approximation for truncation after 20 terms. The corresponding relative error for (47) is rather low, as it is typically $\epsilon_{r}<10^{-5}$. Likewise, (56) is shown to be relatively tight while the overall involved relative error is proportional to the value of $r$ and is $\epsilon_{r}<10^{-6}$ when $r<1$. Figure 2a also illustrates the behaviour of (38), (46) and (47) along with respective numerical results while (56) is depicted in Fig. 2b for three different scenarios. It is evident that the analytical results match their numerical counterparts in all cases, which indicates the accuracy of the proposed expressions.

\section{New Closed-Form Results FOR THE RICE $I e-$ FUNCTION}

Definition 3: For $x \in \mathbb{R}^{+}$and $0 \leq k \leq 1$, Rice Ie-function is defined by the following finite integral representation [18], [62],

$$
I e(k, x) \triangleq \int_{0}^{x} e^{-t} I_{0}(k t) \mathrm{d} t .
$$

The above representation can be alternatively expressed in trigonometric form as [18],

$$
I e(k, x)=\frac{1}{\sqrt{1-k^{2}}}-\frac{1}{\pi} \int_{0}^{\pi} \frac{e^{-x(1-k \cos \theta)}}{1-k \cos \theta} \mathrm{d} \theta .
$$

An analytic expression in terms of the Marcum $Q$-function as well as two alternative series representations were reported in [18] and [62]. These series are infinite and are expressed in terms of the modified Struve function, $L_{n}($.$) , and the$ $\Gamma(),. I_{n}($.$) functions, respectively [63]. Furthermore, they were$

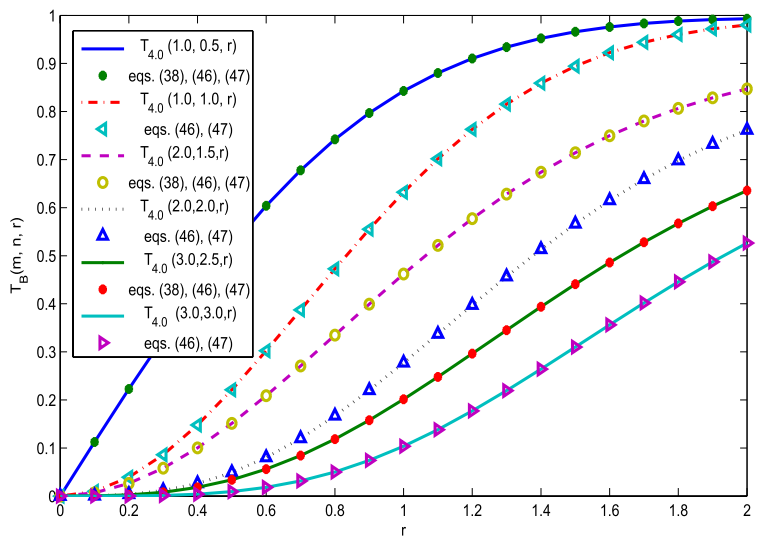

(a)

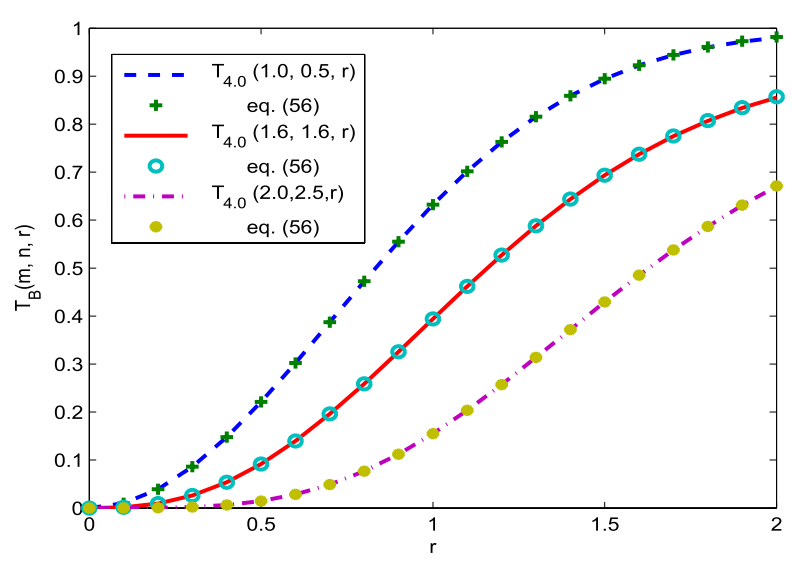

(b)

Fig. 2. Behaviour and accuracy of the incomplete Toronto function in equations (38), (46) (47) \& (56). (a) $T_{B}(m, n, r)$ in (38), (46) \& (47). (b) $T_{B}(m, n, r)$ in (56).

shown to be complementary to each-other as [18, eq. (2)] converges relatively quickly when $x \sqrt{1-k^{2}}$ is large and $k x$ is small, whereas [18, eq. (3)] converges relatively quickly when $x \sqrt{1-k^{2}}$ is small and $k x$ is large. Therefore, it appears that utilizing these series is rather inconvenient both analytically and numerically for the following reasons: $i$ ) two infinite series are required for computing the $I e(k, x)$ function; $i i)$ the $L_{n}($.) function is neither tabulated nor built-in in widely used mathematical software packages.

\section{A. Closed-Form Upper and Lower Bounds}

The lack of simple expressions for the $I e(k, x)$ function constitutes the derivation of tight upper and lower bounds advantageous. To this end, it is critical to primarily express $I e(k, x)$ function alternatively.

Lemma 5: For $x \in \mathbb{R}^{+}$and $0 \leq k \leq 1$, the following analytic representation is valid,

$$
I e(k, x)=1-e^{-x} I_{0}(k x)+k \int_{0}^{x} e^{-t} I_{1}(k t) \mathrm{d} t .
$$


Proof: By integrating (57) by parts one obtains,

$$
I e(k, x)=\left[\int_{0}^{x} e^{-t} \mathrm{~d} t\right] I_{0}(k t)-\int_{0}^{x}\left[\int_{0}^{x} e^{-t} \mathrm{~d} t\right] \frac{\mathrm{d} I_{0}(k t)}{\mathrm{d} t} \mathrm{~d} t .
$$

Based on the basic principles of integration it follows straightforwardly that,

$$
\int_{0}^{x} e^{-t} \mathrm{~d} t=1-e^{-x}
$$

By also recalling that

$$
\frac{\mathrm{d}}{\mathrm{d} x} I_{n}(k x)=\frac{k}{2}\left[I_{n-1}(k x)+I_{n+1}(k x)\right]
$$

and

$$
I_{-1}(x) \triangleq I_{1}(x)
$$

it follows that

$$
\frac{\mathrm{d} I_{0}(k t)}{\mathrm{d} t}=k I_{1}(k t) .
$$

Therefore, by substituting accordingly in (60) one obtains (59), which completes the proof.

Capitalizing on Lemma 5, we derive closed-form upper and lower bounds for the $\operatorname{Ie}(k, x)$ function.

Theorem 5: For $x \in \mathbb{R}^{+}$and $0 \leq k \leq 1$, the following inequalities can serve as upper and lower bounds for the Rice Ie-function,

$$
\begin{aligned}
I e(k, x)<1 & +\sqrt{\frac{k}{2(1-k)}}+\frac{\sqrt{2 k} Q(\sqrt{2 x} \sqrt{1+k})}{\sqrt{1+k}} \\
& -\frac{I_{0}(k x)}{e^{x}}-\sqrt{\frac{k}{2(1+k)}}-\frac{\sqrt{2 k} Q(\sqrt{2 x} \sqrt{1-k})}{\sqrt{1-k}}
\end{aligned}
$$

and ${ }^{2}$

$$
I e(k, x)>\frac{2 Q(b+a)+2 Q(b-a)-e^{-x} I_{0}(k x)-1}{\sqrt{1-k^{2}}}
$$

where

$$
a=\sqrt{x} \sqrt{1+\sqrt{1-k^{2}}}
$$

and

$$
b=\sqrt{x} \sqrt{1-\sqrt{1-k^{2}}} .
$$

Proof: The proof is provided in Appendix F.

Remark 6: The authors in [46] derived closed-form bounds for the $Q_{m}(a, b)$ function. By performing the necessary change of variables and substituting accordingly in [62, eq. (2c)], an alternative closed-form upper bound can be obtained. However, the algebraic representation of such a bound is significantly less compact and less convenient than (65) both analytically and numerically. Likewise, a lower bound for the Ie $(k, x)$ function could be theoretically derived by following the same methodology as in Theorem 2. Nevertheless, this approach leads to an integral representation whose analytic solution is divergent.

\footnotetext{
${ }^{2}$ Eq. (65) can be also expressed in terms of the error function, erf $(x)$, and the complementary error function, $\operatorname{erfc}(x)=1-\operatorname{erf}(x)$ with the aid of the identities: $Q(x) \triangleq \frac{1}{2} \operatorname{erfc}\left(\frac{x}{\sqrt{2}}\right)=\frac{1}{2}-\frac{1}{2} \operatorname{erf}\left(\frac{x}{\sqrt{2}}\right)$.
}

\section{B. A Closed-Form Expression in Terms of Humbert Function}

Theorem 6: For $0 \leq k \leq 1$ and $x \in \mathbb{R}^{+}$, the following expression is valid for the Ie $(k, x)$ function,

$$
I e(k, x)=\frac{1}{\sqrt{1-k^{2}}}-\frac{e^{-(1+k) x}}{1+k} \Phi_{1}\left(\frac{1}{2}, 1,1, \frac{2 k}{1+k}, 2 k x\right)
$$

where $\Phi_{1}(a, b, c, x, y)$ denotes the Humbert series, or confluent Appell function of the first kind [63], [84], [85].

Proof: The proof is provided in Appendix G.

\section{A Simple Polynomial Representation}

Similar to the case of $Q_{m, n}(a, b)$ and $T_{B}(m, n, r)$ functions, a simple representation for the Rice $I e$-function is advantageous for cases that parameter generality and/or algebraic simplicity are required.

Proposition 5: For $x, k \in \mathbb{R}^{+}$and $0 \leq k \leq 1$, the following polynomial approximation is valid for the $\operatorname{Ie}(k, x)$ function,

$$
I e(k, x) \simeq \sum_{l=0}^{L} \frac{\Gamma(L+l) L^{1-2 l} k^{2 l} \gamma(1+2 l, x)}{l ! \Gamma(L-l+1) \Gamma(l+1) 2^{2 l}}
$$

which as $L \rightarrow \infty$, it becomes an exact infinite series representation,

$$
I e(k, x)=\sum_{l=0}^{\infty} \frac{k^{2 l} \gamma(1+2 l, x)}{l ! \Gamma(l+1) 2^{2 l}} .
$$

Proof: The proof follows immediately from Proposition 1 and Proposition 3.

\section{A Closed-Form Upper Bound for the Truncation Error}

The precise accuracy of (70) can be quantified by an upper bound for the truncation error.

Lemma 6: For $k, x \in \mathbb{R}^{+}$and $0 \leq k \leq 1$, the following closed-form inequality holds for the truncation error in (70),

$$
\begin{aligned}
\epsilon_{t}< & +\sqrt{\frac{k}{2(1-k)}}\{1-2 Q(\sqrt{2 x} \sqrt{1-k})\} \\
& -e^{-x} I_{0}(k x)-\sum_{l=0}^{L} \frac{\Gamma(L+l) k^{2 l} \gamma(1+2 l, x)}{l ! l ! L^{2 l-1}(L-l) ! 2^{2 l}} \\
& -\sqrt{\frac{k}{2(1+k)}}\{1-2 Q(\sqrt{2 x} \sqrt{1+k})\} .
\end{aligned}
$$

Proof: When (70) is truncated after $L$ terms, the corresponding truncation error is given by,

$$
\begin{aligned}
\epsilon_{t} & =\sum_{l=L+1}^{\infty} \frac{\Gamma(L+l) k^{2 l} \gamma(1+2 l, x)}{l ! L^{2 l-1}(L-l) ! l ! 2^{2 l}} \\
& =\underbrace{\sum_{l=0}^{\infty} \frac{\gamma(1+2 l, x)}{l ! l ! 2^{2 l} k^{-2 l}}}_{\mathcal{I}_{9}}-\sum_{l=0}^{L} \frac{\Gamma(L+l) k^{2 l} \gamma(1+2 l, x)}{l !(L-l) ! l ! L^{2 l-1} 2^{2 l}} .
\end{aligned}
$$

Since

$$
\mathcal{I}_{9}=\operatorname{Ie}(k, x)
$$


TABLE III

ACCURACY OF PROPOSED EXPRESSIONS FOR THE $I e(k, x)$ FUNCTION

\begin{tabular}{|c|c|c|c|c|c|}
\hline FUNCTION & EXACT & Eq. (65) & Eq. (66) & Eq. (69) & Eq. (70) \\
\hline \hline$I e(0.1,0.1)$ & 0.0952 & 0.0952 & 0.0631 & 0.0952 & 0.0952 \\
\hline$I e(0.1,0.4)$ & 0.3297 & 0.3328 & 0.2829 & 0.3297 & 0.3297 \\
\hline$I e(0.4,0.4)$ & 0.3303 & 0.3526 & 0.1384 & 0.3303 & 0.3303 \\
\hline$I e(0.6,0.4)$ & 0.3311 & 0.3696 & 0.0079 & 0.3311 & 0.3311 \\
\hline$I e(0.6,0.8)$ & 0.5993 & 0.6380 & 0.2630 & 0.5993 & 0.5993 \\
\hline$I e(0.8,0.9)$ & 0.6139 & 0.7400 & 0.1110 & 0.6139 & 0.6139 \\
\hline
\end{tabular}

equation (73) can be equivalently expressed as follows,

$$
\epsilon_{t}=I e(k, x)-\sum_{l=0}^{L} \frac{\Gamma(L+l) L^{1-2 l} k^{2 l} \gamma(1+2 l, x)}{l ! \Gamma(L-l+1) \Gamma(l+1) 2^{2 l}} .
$$

The $I e(k, x)$ can be upper bounded with the aid of the closedform upper bound in (65). As a result, the inequality in (72) is deduced, which completes the proof.

Remark 7: By omitting the terms

$$
\frac{\Gamma(L+l) L^{1-2 l}}{\Gamma(L-l+1)}
$$

from the finite series term of (72), a similar closed-form upper bound is deduced for (71), namely,

$$
\begin{aligned}
\epsilon_{t}< & 1+\sqrt{\frac{k}{2(1-k)}}\{1-2 Q(\sqrt{2 x} \sqrt{1-k})\}-e^{-x} I_{0}(k x) \\
& -\sum_{l=0}^{L} \frac{\gamma(1+2 l, x)}{l ! l ! L^{2 l-1} 2^{2 l}}-\sqrt{\frac{k}{2(1+k)}}\{1-2 Q(\sqrt{2 x} \sqrt{1+k})\}
\end{aligned}
$$

which is also tight.

Table III illustrates the behaviour of the derived expressions for the $I e(k, x)$ function. The proposed bounds are fairly tight for different values of $k$ and $x$ while it is clear that (69) and (70) are in excellent agreement with the respective exact numerical results.

Figures $3 \mathrm{a}$ and $3 \mathrm{~b}$ also illustrate the behaviour of the bounds in (65) and (66) versus $x$ and $k$, respectively. It is observed that the upper bound becomes tighter for small values of $x$ while for higher values of $x$ the lower bound appears to be tighter. Overall, it is observed that the lower bound is significantly tighter than the upper bound. This is also evident by Fig. 3c which indicates that the lower bound in (66) becomes a remarkably accurate approximation to $\operatorname{Ie}(k, x)$ for large values of $x$ as $\epsilon_{r}<10^{-10}$ when $0 \leq k \leq 0.6$ and $\epsilon_{r}<10^{-5}$ when $0.6<k \leq 1$. This figure also depicts the behaviour of the closed-form expression in (69) as well as the polynomial approximation in (70) which is shown to be in excellent agreement with the numerical results. This was achieved for truncation after 20 terms which results to an involved error $\epsilon_{r}<10^{-8}$.

\section{NEW EXPRESSIONS FOR THE INCOMPLETE LIPSCHITZ-HANKEL INTEGRALS}

Definition 4: For $m, a, n, x \in \mathbb{R}^{+}$, the general incomplete Lipschitz Hankel Integral is defined by the following

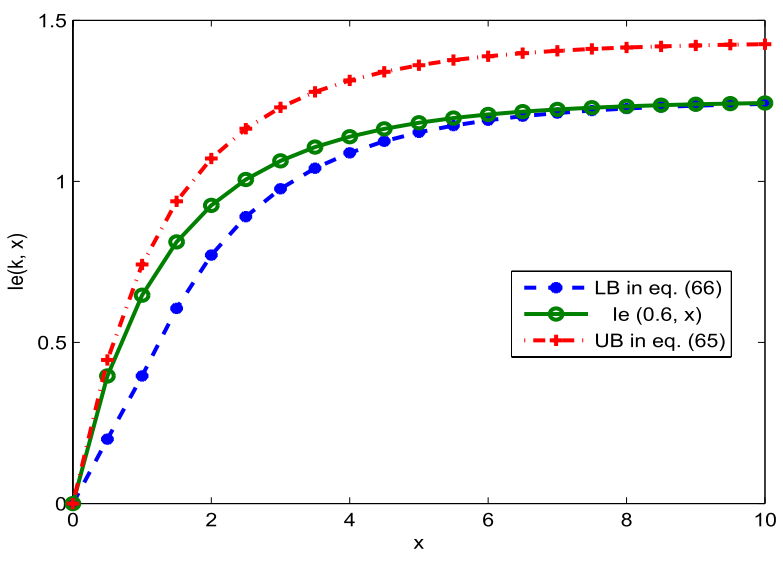

(a)

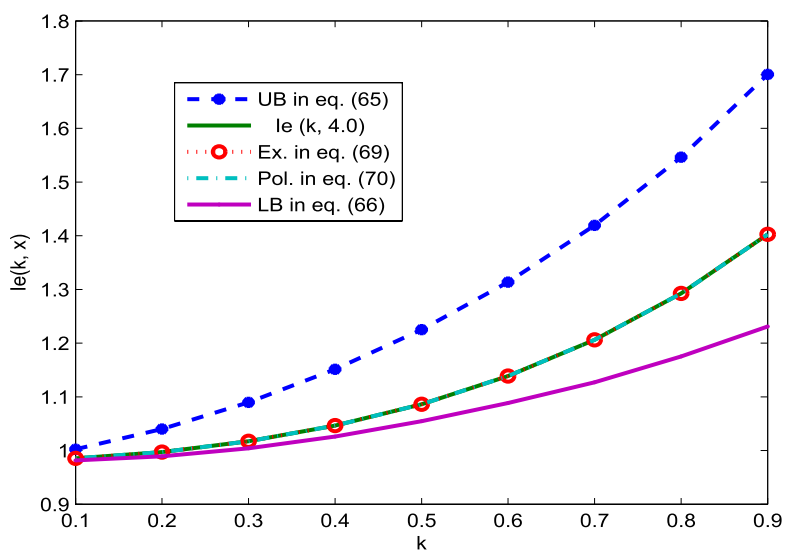

(b)

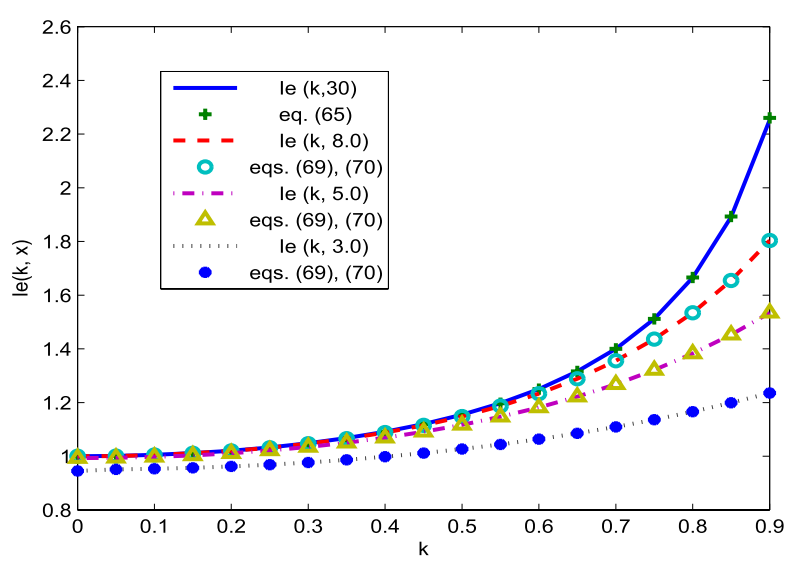

(c)

Fig. 3. Behaviour of the $I e(k, x)$ bounds, series, closed-form and approximation. (a) $I e(k, x)$ in (65) and (66) vs $x$. (b) $I e(k, x)$ in (65), (66), (69) and (70) vs $k$. (c) $I e(k, x)$ in (65), (69) and (70) vs $k$.

non-infinite integral representation,

$$
Z e_{m, n}(x ; a) \triangleq \int_{0}^{x} y^{m} e^{-a y} Z_{n}(y) \mathrm{d} y
$$

where $Z_{n}(x)$ can be one of the cylindrical functions $J_{n}(x)$, $I_{n}(x), Y_{n}(x), K_{n}(x), H_{n}^{1}(x)$ or $H_{n}^{2}(x),[13]$, [20]. 
An alternative representation for the $I_{n}(x)$ based ILHIs was reported in [64], namely,

$$
\begin{aligned}
I e_{m, n}(x ; a)= & A_{m, n}^{0}(a)+e^{-a x} \sum_{i=0}^{m} \sum_{j=0}^{n+1} \frac{B_{m, n}^{i, j}(a)}{\left[x^{i} I_{j}(x)\right]^{-1}} \\
& +\frac{Q_{1}\left(\sqrt{\frac{x}{a+\sqrt{a^{2}-1}}}, \sqrt{x} \sqrt{a+\sqrt{a^{2}-1}}\right)}{\left[A_{m, n}^{1}(a)\right]^{-1}}
\end{aligned}
$$

where the set of coefficients $A_{m, n}^{l}(a)$ and $B_{m, n}^{i, j}(a)$ can be obtained recursively, [64]. The above expression was employed in analytical investigations on error rate of MIMO systems under imperfect channel state information. Nevertheless, its algebraic representation is relatively inconvenient and laborious to handle analytically and numerically.

\section{A. Special Cases}

A closed-form expression for $I e_{m, n}(x ; a)$ can be derived for the special case that $m$ and $n$ are positive half-integers.

Theorem 7: For $a \in \mathbb{R}, x \in \mathbb{R}^{+}, m \pm 0.5 \in \mathbb{N}, n \pm 0.5 \in \mathbb{N}$ and $m \geq n$, the following closed-form expression holds for the $I e_{m, n}(x ; a)$ integrals,

$$
\begin{aligned}
I e_{m, n}(x ; a)= & \sum_{k=0}^{n-\frac{1}{2}} \frac{\Gamma\left(n+k+\frac{1}{2}\right)}{\sqrt{\pi} k ! 2^{k+\frac{1}{2}} \Gamma\left(n-k+\frac{1}{2}\right)} \\
& \times\left\{\frac{(-1)^{k} \gamma\left(m-k+\frac{1}{2},(a-1) x\right)}{(a-1)^{m-k+\frac{1}{2}}}\right. \\
& \left.+\frac{(-1)^{n+\frac{1}{2}} \gamma\left(m-k+\frac{1}{2},(a+1) x\right)}{(a+1)^{m-k+\frac{1}{2}}}\right\}
\end{aligned}
$$

Proof: The proof is provided in Appendix H.

Likewise, a closed-form expression is derived for the special case that the sum of the indices $m$ and $n$ is a positive integer.

Theorem 8: For $m \in \mathbb{R}, n \in \mathbb{R}, x \in \mathbb{R}^{+}, a>1$ and $m+n \in \mathbb{N}$, the following closed-form expression is valid for the $I e_{m, n}(x ; a)$ integrals,

$$
\begin{aligned}
I e_{m, n}(x ; a)= & \frac{\Gamma(m+n+1)}{2^{n} n ! a^{m+n+1}} \\
& \times{ }_{2} F_{1}\left(\frac{m+n+1}{2}, \frac{m+n}{2}+1 ; 1+n ; \frac{1}{a^{2}}\right) \\
& -\sum_{l=0}^{m+n}\left(\begin{array}{c}
m+n \\
l
\end{array}\right) \frac{l ! x^{m+n-l} e^{-x(1+a)}}{(1+a)^{l+1} 2^{n} n !} \\
& \times \Phi_{1}\left(n+\frac{1}{2}, 1+l, 1+2 n ; \frac{2}{1+a}, 2 x\right)
\end{aligned}
$$

where ${ }_{2} F_{1}(a, b ; c ; x)$ denotes the Gauss hypergeometric function [63].

Proof: The proof is provided in Appendix I.

In the same context, simple closed-form expressions can be derived for the specific cases that $m=-n$ and $m=n=0$.

Theorem 9: For $m \in \mathbb{R}, n \in \mathbb{R}, x \in \mathbb{R}^{+}, a>1$ and $m=-n$, the following closed-form expression is valid for the $I e_{m, n}(x ; a)$ integrals,

$$
\begin{aligned}
I e_{-n, n}(x ; a)= & \frac{{ } F_{1}\left(n+\frac{1}{2}, 1 ; 1+2 n ; \frac{2}{1+a}\right)}{(1+a) n ! 2^{n}} \\
& -\frac{\Phi_{1}\left(n+\frac{1}{2}, 1,1+2 n ; \frac{2}{1+a}, 2 x\right)}{2^{n}(1+a) \Gamma(n+1) e^{x(1+a)}}
\end{aligned}
$$

which for the specific case that $m=n=0$ can be expressed as follows,

$$
\begin{aligned}
I e_{m=0, n=0}(x ; a) & =I e_{0,0}(x ; a) \\
& =\frac{Q_{1}(\mathrm{~b}, \mathrm{c})-Q_{1}(\mathrm{c}, \mathrm{b})}{\sqrt{(a+1)(a-1)}}
\end{aligned}
$$

where

$$
\mathrm{b}=\sqrt{x} \sqrt{a+\sqrt{(a+1)(a-1)}}
$$

and

$$
\mathrm{c}=\sqrt{x} \sqrt{a-\sqrt{(a+1)(a-1)}} .
$$

Proof: The proof is provided in Appendix J.

\section{B. Closed-Form Upper and Lower Bounds}

Capitalizing on the derived closed-form expression for the $I e_{m, n}(x ; a)$ integrals in Theorem 7 , tight closed-form upper and lower bounds can be readily deduced.

Lemma 7: For $m, n, x, a \in \mathbb{R}^{+}$and $m \geq n$, the following inequalities can serve as upper and lower bounds to the $I_{n}(x)$ based incomplete Lipschitz Hankel integrals,

$$
I e_{m, n}(x ; a) \leq I e_{\lceil m\rceil_{0.5},\lceil n\rceil_{0.5}}(x ; a)
$$

and

$$
I e_{m, n}(x ; a) \geq I e_{\lfloor m\rfloor_{0.5},\lfloor n\rfloor_{0.5}}(x ; a) .
$$

Proof: The $I e_{m, n}(x ; a)$ integrals are monotonically increasing w.r.t $m$ and monotonically decreasing w.r.t $n$. By recalling the two half-integer rounding operators in [46, eq. (18)] as well as that (80) holds for $m \pm 0.5 \in \mathbb{N}$ and

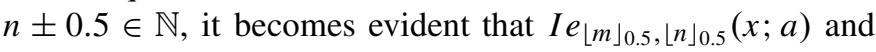
$I e_{\lceil m\rceil_{0.5},\lceil n\rceil_{0.5}}(x ; a)$ can be expressed in closed-form for any value of $m, n, r$ and $x$. As a result, equations (87) and (88) are deduced and thus, completing the proof.

\section{A Simple Polynomial Representation}

The proposed expressions for the $I e_{m, n}(x ; a)$ integrals can be useful for applications related to wireless communications. However, a simpler and more general analytic expression is additionally necessary for scenarios that require unrestricted parameters and/or rather simple algebraic representation.

Proposition 6: For $a, m, n \in \mathbb{R}$ and $x \in \mathbb{R}^{+}$, the following expression holds for the $I e_{m, n}(x ; a)$ integrals,

$$
I e_{m, n}(x ; a) \simeq \sum_{l=0}^{L} \frac{\Gamma(L+l) L^{1-2 l} \gamma(m+n+2 l+1, a x)}{l !(L-l) !(n+l) ! 2^{n+2 l} a^{m+n+2 l+1}}
$$


which as $L \rightarrow \infty$ it reduces to the following exact infinite series representation,

$$
I e_{m, n}(x ; a)=\sum_{l=0}^{\infty} \frac{\gamma(m+n+2 l+1, a x)}{l !(n+l) ! 2^{n+2 l} a^{m+n+2 l+1}} .
$$

Proof: The proof is provided in Appendix K.

\section{A Closed-Form Upper Bound for the Truncation Error}

Lemma 8: For $a, m, n \in \mathbb{R}$ and $x \in \mathbb{R}^{+}$, the following inequality holds as an upper bound for the truncation error of (89),

$$
\begin{aligned}
\epsilon_{t} \leq & \sum_{k=0}^{\lceil n\rceil_{0.5}-\frac{1}{2}} \frac{2^{-k-\frac{1}{2}} \Gamma\left(\lceil n\rceil_{0.5}+k+\frac{1}{2}\right)}{\sqrt{\pi} k ! \Gamma\left(\lceil n\rceil_{0.5}-k+\frac{1}{2}\right)} \\
& \times\left\{\frac{(-1)^{k} \gamma\left(m-k+\frac{1}{2},(a-1) x\right)}{(a-1)^{m-k+\frac{1}{2}}}\right. \\
& \left.+\frac{(-1)^{\lceil n\rceil_{0.5}+\frac{1}{2}} \gamma\left(m-k+\frac{1}{2},(a+1) x\right)}{(a+1)^{m-k+\frac{1}{2}}}\right\} \\
& -\sum_{l=0}^{L} \frac{\Gamma(L+l) L^{1-2 l} \gamma(m+n+2 l+1, a x)}{l !(L-l) !(n+l) ! 2^{n+2 l} a^{m+n+2 l+1}} .
\end{aligned}
$$

Proof: Since (89) reduces to (90) as $L \rightarrow \infty$, the corresponding truncation error is given by,

$$
\begin{aligned}
\epsilon_{t}= & \underbrace{\sum_{l=0}^{\infty} \frac{\gamma(m+n+2 l+1, a x)}{l !(n+l) ! 2^{n+2 l} a^{m+n+2 l+1}}}_{\mathcal{I}_{10}} \\
& -\sum_{l=0}^{L} \frac{\Gamma(L+l) L^{1-2 l} \gamma(m+n+2 l+1, a x)}{l !(L-l) !(n+l) ! 2^{n+2 l} a^{m+n+2 l+1}} .
\end{aligned}
$$

Notably,

$$
\mathcal{I}_{10}=I e_{m, n}(x ; a)
$$

while $I e_{m, n}(x ; a)$ can be upper bounded using $I e_{\lceil m\rceil_{0.5},[n\rceil_{0.5}}(x ; a)$. As a result, by substituting (80) into (92) one obtains (94), which completes the proof.

Remark 8: By omitting the terms

$$
\frac{\Gamma(L+l) L^{1-2 l}}{(L-l) !}
$$

in (94), a similar upper bound is also deduced for (90), namely,

$$
\begin{aligned}
\epsilon_{t} \leq & \sum_{k=0}^{\lceil n\rceil_{0.5}-\frac{1}{2}} \frac{2^{-k-\frac{1}{2}} \Gamma\left(\lceil n\rceil_{0.5}+k+\frac{1}{2}\right)}{\sqrt{\pi} k ! \Gamma\left(\lceil n\rceil_{0.5}-k+\frac{1}{2}\right)} \\
& \times\left\{\frac{(-1)^{k} \gamma\left(m-k+\frac{1}{2},(a-1) x\right)}{(a-1)^{m-k+\frac{1}{2}}}\right. \\
& \left.+\frac{(-1)^{\lceil n\rceil_{0.5}+\frac{1}{2}} \gamma\left(m-k+\frac{1}{2},(a+1) x\right)}{(a+1)^{m-k+\frac{1}{2}}}\right\} \\
& -\sum_{l=0}^{L} \frac{\gamma(m+n+2 l+1, a x)}{l !(n+l) ! 2^{n+2 l} a^{m+n+2 l+1}} .
\end{aligned}
$$

which is also rather tight.

\section{E. A Tight Closed-Form Upper Bound and Approximation}

The algebraic representation of the $I e_{m, n}(x ; a)$ integrals allows the derivation of a simple upper bound which in certain range of values becomes an accurate approximation.

Proposition 7: For $m, n, a, x \in \mathbb{R}^{+}$and $x, a>m, n$, the following inequality is valid for the ILHIs,

$$
I e_{m, n}(x ; a) \leq \frac{(n+1)_{m 2} F_{1}\left(\frac{m+n+1}{2}, \frac{m+n}{2}+1 ; n+1 ; \frac{1}{a^{2}}\right)}{a^{m+n+1} 2^{n}}
$$

which for $a>3$ and , $x>3$ becomes an accurate closed-form approximation.

Proof: The $\gamma(a, x)$ function can be upper bounded with the aid of the following $\Gamma(a)$ function property,

$$
\Gamma(a)=\gamma(a, x=\infty) .
$$

To this effect, the $I e_{m, n}(x ; a)$ integrals can be upper bounded as follows:

$$
I e_{m, n}(x ; a) \leq \sum_{l=0}^{L} \frac{\Gamma(L+l) L^{1-2 l} \Gamma(m+n+2 l+1)}{l !(L-l) !(n+l) ! 2^{n+2 l} a^{m+n+2 l+1}} .
$$

As $L \rightarrow \infty$ and recalling that $x !=\Gamma(x+1)$ and $\Gamma(a, n)=$ $(a)_{n} \Gamma(a)$ it immediately follows that,

$$
I e_{m, n}(x ; a) \leq \sum_{l=0}^{\infty} \frac{(m+n+1)_{2 l} \Gamma(m+n+1) 2^{-2 l}}{l !(n+1)_{l} \Gamma(n+1) 2^{n} a^{m+n+2 l+1}} .
$$

Importantly, with the aid of the identity,

$$
(2 x)_{2 l} \triangleq 2^{2 l}(x)_{l}(x+0.5)_{l}
$$

equation (98) can be also expressed as,

$$
I e_{m, n}(x ; a) \leq \frac{(m+n) !}{(n) ! a^{m+n+1}} \sum_{l=0}^{\infty} \frac{\left(\frac{m+n+1}{2}\right)_{l}\left(\frac{m+n}{2}+1\right)_{l}}{l !(n+1){ }_{l} 2^{n+2 l} a^{2 l} 2^{-2 l}} .
$$

The above series can be expressed in closed-form in terms of the Gaussian hypergeometric function ${ }_{2} F_{1}(a, b ; c ; x)$. Hence, by substituting in (100) and performing some basic algebraic manipulations (95) is deduced thus, completing the proof. $\square$

The accuracy of the derived analytic expressions for the $I e_{m, n}(x ; a)$ integrals is depicted in Table IV (top of the next page) along with respective results from numerical integrations. One can notice the excellent agreement between analytical and numerical results while the proposed upper bound and approximation appear to be quite accurate. Specifically, truncating (89) after 30 terms and for $a<2$ yields a relative error of $\epsilon_{r}<10^{-4}$. It is also noticed that the tight upper bound for small values of $a$ becomes an accurate approximation as $a$ increases. This is additionally evident by the involved relative error which can be as low as $\epsilon_{r}<10^{-9}$.

In the same context, the accuracy of the proposed polynomial approximations for the above functions and integrals is depicted in Table $\mathrm{V}$ (top of the next page) in terms of the involved relative error. Evidently, the value of $\epsilon_{r}$ is rather low for numerous different parametric scenarios which indicates the overall high accuracy of the proposed analytic expressions. 
TABLE IV

ACCURACY OF PROPOSED EXPRESSIONS FOR THE $I e_{m, n}(x ; a)$ INTEGRALS

\begin{tabular}{|c|c|c|c|c|}
\hline FUNCTION & EXACT & Eqs. (80), (81), (82), (83) & Eq. (89) & Eq. (95) \\
\hline \hline$I e_{0,0}(3.2 ; 1.7)$ & 0.6974 & $\mathrm{n} / \mathrm{a}, 0.6974, \mathrm{n} / \mathrm{a}, 0.6974$ & 0.6974 & 0.7274 \\
\hline$I e_{0,0}(3.2 ; 2.7)$ & 0.3982 & $\mathrm{n} / \mathrm{a}, 0.3982, \mathrm{n} / \mathrm{a}, 0.3982$ & 0.3982 & 0.3987 \\
\hline$I e_{0.5,0.5}(3.2 ; 1.7)$ & 0.3615 & $0.3615,0.3615, \mathrm{n} / \mathrm{a}, \mathrm{n} / \mathrm{a}$ & 0.3615 & 0.4222 \\
\hline$I e_{0.5,0.5}(3.2 ; 2.7)$ & 0.1258 & $0.1258,0.1258, \mathrm{n} / \mathrm{a}, \mathrm{n} / \mathrm{a}$ & 0.1258 & 0.1268 \\
\hline$I e_{-0.5,0.5}(3.2 ; 1.7)$ & 0.5245 & $\mathrm{n} / \mathrm{a}, 0.5245,0.5245, \mathrm{n} / \mathrm{a}$ & 0.5245 & 0.5385 \\
\hline$I e_{-0.5,0.5}(3.2 ; 2.7)$ & 0.3000 & n/a, $0.3000,0.3000, \mathrm{n} / \mathrm{a}$ & 0.3000 & 0.3103 \\
\hline
\end{tabular}

TABLE V

Absolute Relative Error for All Proposed Series Representations

\begin{tabular}{|c|c||c|c|}
\hline FUNCTION & $\mathrm{n}=30$ & FUNCTION & $\mathrm{n}=30$ \\
\hline \hline$Q_{1.1,0.8}(1.7,1.4)$ & $5.0 \times 10^{-13}$ & $I e_{1.1,0.8}(1.7 ; 1.4)$ & $4.0 \times 10^{-10}$ \\
\hline$Q_{1.1,1.4}(1.9,1.2)$ & $9.7 \times 10^{-12}$ & $I e_{1.1,1.4}(1.9 ; 1.2)$ & $9.4 \times 10^{-11}$ \\
\hline$Q_{2.2,0.9}(2.1,1.9)$ & $1.9 \times 10^{-13}$ & $I e_{2.2,0.9}(2.1 ; 1.9)$ & $3.0 \times 10^{-10}$ \\
\hline$Q_{0.9,1.2}(0.6,0.9)$ & $7.3 \times 10^{-13}$ & $I e_{0.9,1.2}(0.6 ; 0.9)$ & $9.1 \times 10^{-11}$ \\
\hline$Q_{1.7,1.7}(0.3,0.2)$ & $1.8 \times 10^{-13}$ & $I e_{1.7,1.7}(0.3 ; 0.2)$ & $1.5 \times 10^{-6}$ \\
\hline \hline$T_{3}(1.8,0.9,0.7)$ & $7.5 \times 10^{-10}$ & $\operatorname{Ie}(0.3,1.8)$ & $1.2 \times 10^{-15}$ \\
\hline$T_{3}(1.1,1.9,1.2)$ & $9.8 \times 10^{-9}$ & $I e(0.3,3.1)$ & $1.5 \times 10^{-15}$ \\
\hline$T_{4}(1.3,1.3,1.9)$ & $2.1 \times 10^{-9}$ & $I e(0.9,1.2)$ & $1.3 \times 10^{-15}$ \\
\hline$T_{4}(2.7,2.7,2.7)$ & $7.3 \times 10^{-12}$ & $I e(0.9,4.8)$ & $1.4 \times 10^{-15}$ \\
\hline
\end{tabular}

The behaviour of the analytic expressions in (80), (81), (82), (83) \& (89) is illustrated in Fig. 4a along with respective results from numerical integrations. One can notice the excellent agreement between analytical and numerical results. For (89), this is achieved by truncating the series after 30 terms which corresponds to a relative error of $\epsilon_{r}<$ $10^{-4}$ when $a<2$. Likewise, the accuracy of (100) is illustrated in Fig. 4b where it is observed that the tight upper bound for small values of $a$ becomes an accurate approximation as $a$ increases asymptotically. This is also evident by the involved relative error which can be as low as $\epsilon_{r}<10^{-9}$.

\section{Closed-Form Expressions for Special CASES OF THE KAMPE DE FERIET AND THE HUMBERT $\Phi_{1}$ FUNCTIONS}

The previous Sections were devoted to the derivation of novel analytic expressions for the $Q_{m, n}(a, b), T_{B}(m, n, r)$, $I e(k, x)$ functions and the $I e_{m, n}(x ; a)$ integrals. Capitalizing on the offered analytic results, useful closed-form expression can be readily deduced for special cases of the $\mathrm{KdF}$ and Humbert $\Phi_{1}$ special functions. It is noted here that these functions are rather general and particularly the $\mathrm{KdF}$ can represent the vast majority of special functions. As a result, relating expressions are rather necessary in unified representations of different special functions that are used in digital communications.

Corollary 1: For $x, y \in \mathbb{R}^{+}$and $a>-\frac{1}{2}, b>-1$, the following closed-form expression is valid,

$$
\begin{aligned}
F_{1,1}^{1,0}\left(\begin{array}{l}
a:-,-: \\
a+1: b,-:
\end{array} x,-y\right) & =\mathcal{F}_{1}(a, a+1, b ; x,-y) \\
& =\frac{a \Gamma(b) T_{\sqrt{y}}\left(2 a-1, b-1, \sqrt{\frac{x}{y}}\right)}{x^{b-a} y^{2 a-b} e^{-\frac{x}{y}}}
\end{aligned}
$$

where $\mathcal{F}_{1}(\cdot)$ denotes the following infinite series representations,

$$
\begin{aligned}
\mathcal{F}_{1}(a, a+1, b ; x,-y) & =\sum_{l=0}^{\infty} \sum_{i=0}^{\infty} \frac{a}{a+l+i} \frac{x^{l}}{l !} \frac{y^{i}}{i !} \\
& =\sum_{l=0}^{\infty} \sum_{i=0}^{\infty} \frac{(a)_{l+i}}{(a+1)_{l+i}(b)_{l}} \frac{x^{l}}{l !} \frac{y^{i}}{i !}
\end{aligned}
$$

Proof: The proof follows from Theorem 4 by setting

$$
a=\frac{m+1}{2}
$$

and $b=n+1, x=r^{2} B^{2}$ and $y=B^{2}$.

Corollary 2: For $a, b \in \mathbb{R}$ and $x, y \in \mathbb{R}^{+}$, the following closed-form expression is valid,

$$
\begin{aligned}
F_{1,1}^{1,0}\left(\begin{array}{l}
a:-,-: \\
a+1: b,-:
\end{array} x,-y\right)= & \mathcal{F}_{1}(a, a+1, b ; x,-y) \\
= & \frac{a \Gamma(a){ }_{1} F_{1}\left(b-a, b,-\frac{x}{y}\right)}{y^{a} e^{-\frac{x}{y}}} \\
& -\frac{a \Gamma(b) Q_{2 a-b, b-1}\left(\sqrt{\frac{2 x}{y}}, \sqrt{2 y}\right)}{y^{a-\frac{b-1}{2}} e^{-\frac{x}{y}} x^{\frac{b-1}{2}} 2^{a-\frac{b+1}{2}}}
\end{aligned}
$$

where $\mathcal{F}_{1}(a, a+1, b ; x,-y)$ is given in (103).

Proof: The proof follows immediately by applying Lemma 2 in Corollary 1.

Likewise, closed-form expressions are deduced for special cases of the Humbert $\Phi_{1}$ function.

Corollary 3: For $a \in \mathbb{R}, y \in \mathbb{R}^{+}$and $-1<x<1$, the following closed-form expression holds,

$$
\begin{aligned}
\Phi_{1}(a, 1,2 a ; x, y)= & { }_{2} F_{1}(a, 1,2 a, x) e^{\frac{y}{x}} \\
& -\frac{2^{a+\frac{1}{2}} \Gamma\left(a+\frac{1}{2}\right)}{x e^{-\frac{y}{x}}} I e_{\frac{1}{2}-a, a-\frac{1}{2}}\left(\frac{y}{2} ; \frac{2}{x}-1\right) .
\end{aligned}
$$




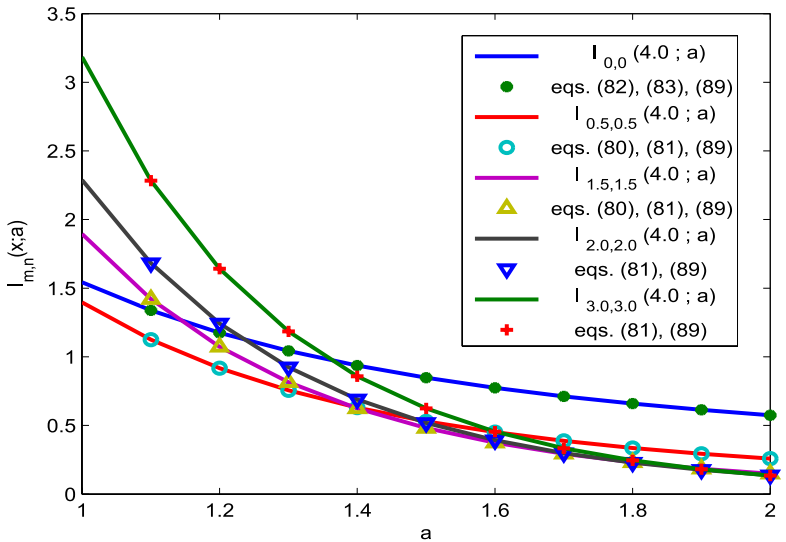

(a)

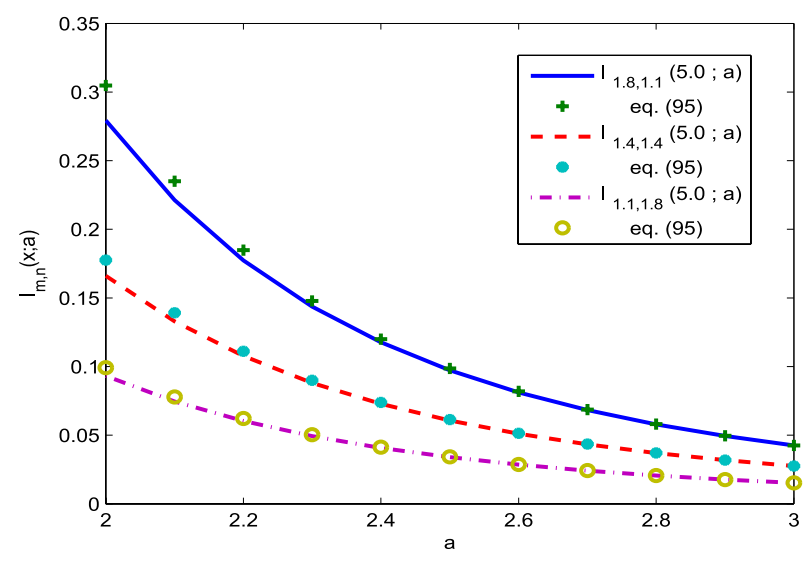

(b)

Fig. 4. Behaviour of the proposed bounds, closed-forms, series and approximation for the $I e_{m, n}(x ; a)$ integrals. (a) $I e_{m, n}(x ; a)$ in (80), (81), (82), (83) \& (89). (b) $I e_{m, n}(x ; a)$ in (95).

Proof: The proof follows immediately from (82) in from Theorem 9 for $a=n+\frac{1}{2}$.

Corollary 4: For $y \in \mathbb{R}^{+}$and $-1<x<1$, the following closed-form expression is valid,

$$
\begin{aligned}
\Phi_{1}\left(\frac{1}{2}, 1,1 ; x, y\right)= & e^{\frac{y}{x}}{ }_{2} F_{1}\left(\frac{1}{2}, 1,1, x\right) \\
& -e^{\frac{y}{x}} \frac{Q_{1}(b, c)-Q_{1}(c, b)}{\sqrt{1-x}}
\end{aligned}
$$

where

$$
b=\sqrt{\frac{y}{x}(1+\sqrt{1-x})-\frac{y}{2}}
$$

and

$$
c=\sqrt{\frac{y}{x}(1-\sqrt{1-x})-\frac{y}{2}} .
$$

Proof: The proof follows from (83) in Theorem 9 by setting $n=0$ and $a=\frac{2}{x}-1$.

\section{APPLICATIONS IN WIRELESS COMMUNICATIONS THEORY}

As already mentioned, the offered analytic results can be particularly useful in the broad area of wireless communications. To this end, they are indicatively employed in deriving analytic expressions for applications relating to digital communications over fading channels. Novel closed-form expressions are derived for the OP over non-linear generalized fading channels that follow the $\alpha-\eta-\mu, \alpha-\lambda-\mu$ and $\alpha-\kappa-\mu$ distributions. These fading models were proposed in [87] and [88] and are distinct for their remarkable flexibility as they have been shown to provide accurate fitting in measurements that correspond to versatile realistic communication scenarios. This is clearly indicated in [87, Fig. 1] while it is also evident by the fact that these models include as special cases the well known $\alpha-\mu, \eta-\mu$ and $\kappa-\mu$ distributions and therefore, the Hoyt, Rice, Weibull, Nakagami $-m$ and Rayleigh distributions [89]-[91]. In addition, closed-form expressions are additionally deduced for specific cases of OP over $\eta-\mu$ and $\lambda-\mu$ fading channels as well as for the truncated channel inversion with fixed rate transmission in both single and multi-antenna systems over Rician fading channels.

\section{A. Outage Probability Over $\alpha-\eta-\mu$ Fading Channels}

The $\alpha-\eta-\mu$ distribution is a particularly flexible fading model that provides accurate characterization of various multipath fading scenarios including modelling of satellite links subject to strong atmospheric scintillation. Furthermore, it constitutes a generalization of $\eta-\mu$ distributions and thus, it includes as special cases the $\eta-\mu, \alpha-\mu$, Hoyt, Nakagami $-m$ and Rayleigh distributions. In terms of physical interpretation of the involved parameters, $\alpha$ denotes the non-linearity parameter which accounts for the non-homogeneous diffuse scattering field, $\mu$ is related to the number of multipath clusters and $\eta$ is the scattered-wave power ratio between the in-phase and quadrature components of each cluster of multipath [87].

Definition 5: For $\alpha, \eta, \mu, \rho \in \mathbb{R}^{+}$, the normalized envelope $P D F$ for the $\alpha-\eta-\mu$ distribution is expressed as,

$$
p_{P}(\rho)=\frac{\alpha(\eta+1)^{\mu+\frac{1}{2}} \sqrt{\pi} \mu^{\mu+\frac{1}{2}} I_{\mu-\frac{1}{2}}\left(\frac{\left(\eta^{2}-1\right) \mu \rho^{\alpha}}{2 \eta}\right)}{\Gamma(\mu) \sqrt{\eta}(\eta-1)^{\mu-\frac{1}{2}} \rho^{1-\alpha\left(\mu+\frac{1}{2}\right)} e^{\frac{(1+\eta)^{2} \mu \rho^{a}}{2 \eta}}} .
$$

Corollary 5: For $\alpha, \eta, \mu, \bar{\gamma}, \gamma_{t h} \in \mathbb{R}^{+}$, the OP over independent and identically distributed (i.i.d) $\alpha-\eta-\mu$ fading channels can be expressed as follows,

$$
\begin{aligned}
P_{\mathrm{out}}= & \frac{\sqrt{\pi} 2^{\mu+\frac{1}{2}} \eta^{\mu}}{\Gamma(\mu)(\eta-1)^{2 \mu}} \\
& \times I e_{\mu+\frac{1}{2}+\frac{4(1-\alpha)}{\alpha^{2}}, \mu-\frac{1}{2}}\left(\frac{\mu\left(\eta^{2}-1\right) \gamma_{t h}^{\alpha / 2}}{2 \eta \bar{\gamma}^{\alpha / 2}} ; \frac{\eta+1}{\eta-1}\right)
\end{aligned}
$$

where $\bar{\gamma}$ and $\gamma_{t h}$ denote the average SNR and the pre-determined SNR threshold, respectively.

Proof: Based on the envelope PDF in (112), the PDF of the corresponding SNR per symbol is given by [87, eq. (1)],

$$
\begin{aligned}
p_{\gamma}(\gamma)= & \frac{\alpha(\eta+1)^{\mu+\frac{1}{2}} \sqrt{\pi} \mu^{\mu+\frac{1}{2}}}{2 \Gamma(\mu) \sqrt{\eta}(\eta-1)^{\mu-\frac{1}{2}}} \frac{\gamma^{\alpha\left(\mu+\frac{1}{2}\right)-1}}{\bar{\gamma}^{\alpha\left(\mu+\frac{1}{2}\right)}} \\
& \times e^{-\frac{(1+\eta)^{2} \mu}{2 \eta} \frac{\gamma^{\alpha / 2}}{\bar{\gamma}^{\alpha / 2}}} I_{\mu-\frac{1}{2}}\left(\frac{\left(\eta^{2}-1\right) \mu}{2 \eta} \frac{\gamma^{\alpha / 2}}{\bar{\gamma}^{\alpha / 2}}\right) .
\end{aligned}
$$


It is also recalled that the OP over fading channels is defined as [23, eq. (1.4)], namely,

$$
P_{\text {out }} \triangleq F\left(\gamma_{t h}\right)=\int_{0}^{\gamma_{t h}} p_{\gamma}(\gamma) \mathrm{d} \gamma
$$

where $F(\gamma)$ is the cumulative distribution function (CDF) of $\gamma$. Thus, by substituting (114) in (115) and after performing necessary change of variables and basic algebraic manipulations yields,

$$
\begin{aligned}
& P_{\text {out }}=\frac{\alpha(\eta+1)^{\mu+\frac{1}{2}} \sqrt{\pi} \mu^{\mu+\frac{1}{2}}}{2 \Gamma(\mu) \sqrt{\eta}(\eta-1)^{\mu-\frac{1}{2}} \bar{\gamma}^{\alpha\left(\mu+\frac{1}{2}\right)}} \\
& \times \int_{0}^{\gamma_{t h}} \frac{\gamma^{\alpha\left(\mu+\frac{1}{2}\right)-1}}{e^{\frac{(1+\eta)^{2} \mu}{2 \eta}} \frac{\gamma^{\alpha / 2}}{\bar{\gamma}^{\alpha / 2}}} I_{\mu-\frac{1}{2}}\left(\frac{\left(\eta^{2}-1\right) \mu}{2 \eta} \frac{\gamma^{\alpha / 2}}{\bar{\gamma}^{\alpha / 2}}\right) \mathrm{d} \gamma .
\end{aligned}
$$

By setting

$$
u=\frac{\left(\eta^{2}-1\right) \mu \gamma^{\alpha / 2}}{\left(2 \eta \bar{\gamma}^{\alpha / 2}\right)}
$$

and carrying out some long but basic algebraic manipulations it follows that,

$$
\begin{aligned}
& P_{\mathrm{out}}=\frac{\sqrt{\pi} \eta^{\mu} 2^{\mu+\frac{1}{2}}}{\Gamma(\mu)(\eta-1)^{2 \mu}} \\
& \times \int_{0}^{\frac{\left(\eta^{2}-1\right) \mu}{2 \eta \eta^{\alpha / 2}} \gamma_{t h}^{\alpha / 2}} u^{\mu+\frac{\alpha^{2}+8(1-\alpha)}{2 \alpha^{2}}} e^{-\frac{\eta+1}{\eta-1} u} I_{\mu-\frac{1}{2}}(u) \mathrm{d} u .
\end{aligned}
$$

Notably, the above integral can be expressed in terms of the ILHIs. As a result (113) is deduced, which completes the proof.

Remark 9: By recalling that

$$
P_{\text {out }} \triangleq F_{\gamma}\left(\gamma_{t h}\right)
$$

it immediately follows from (113) that the CDF of the $\alpha-\eta-\mu$ distribution can be expressed as,

$$
\begin{aligned}
F_{\gamma}(\gamma)= & \frac{\sqrt{\pi} 2^{\mu+\frac{1}{2}} \eta^{\mu}}{\Gamma(\mu)(\eta-1)^{2 \mu}} \\
& \times I e_{\mu+\frac{1}{2}+\frac{4(1-\alpha)}{\alpha^{2}}, \mu-\frac{1}{2}}\left(\frac{\mu\left(\eta^{2}-1\right) \gamma^{\alpha / 2}}{2 \eta \bar{\gamma}^{\alpha / 2}} ; \frac{\eta+1}{\eta-1}\right) .
\end{aligned}
$$

Furthermore, for the specific case that $\alpha=2$, equation (113) yields a closed-form expression for the OP over $\eta-\mu$ fading channels, namely,

$$
P_{\text {out }}=\frac{\sqrt{\pi} 2^{\mu+\frac{1}{2}} \eta^{\mu}}{\Gamma(\mu)(\eta-1)^{2 \mu}} I e_{\mu-\frac{1}{2}, \mu-\frac{1}{2}}\left(\frac{\mu\left(\eta^{2}-1\right) \gamma_{t h}}{2 \eta \bar{\gamma}} ; \frac{\eta+1}{\eta-1}\right)
$$

which is valid for all values of $\eta$ and $\mu$.

\section{B. Outage Probability Over $\alpha-\lambda-\mu$ Fading Channels}

The $\alpha-\lambda-\mu$ distribution has been also proposed as an accurate fading model that represents small scale signal variations. It is closely related to the $\alpha-\eta-\mu$ distribution and is also known as its Format 2 while it includes as special cases the $\lambda-\mu, \alpha-\mu$, Hoyt, Nakagami- $m$ and Rayleigh distributions. In terms of physical interpretation, $\lambda$ is the correlation coefficient between the scattered-wave in-phase and quadrature components of each cluster of multipath while $\alpha$ and $\mu$ denote the non-linearity parameter and the number of multipath clusters, respectively.

Corollary 6: For $-1<\lambda<1$ and $\alpha, \mu, \bar{\gamma}, \gamma_{\text {th }} \in \mathbb{R}^{+}$, the OP over i.i.d $\alpha-\lambda-\mu$ fading channels can be expressed as,

$$
\begin{aligned}
P_{\text {out }}= & \frac{(-1)^{2 \mu} \sqrt{\pi}(1-\lambda)^{\mu}(1+\lambda)^{\mu}}{\Gamma(\mu) 2^{\mu-\frac{1}{2}} \lambda^{2 \mu}} \\
& \times I e_{\mu+\frac{1}{2}+\frac{4(1-\alpha)}{\alpha^{2}}, \mu-\frac{1}{2}}\left(\frac{2 \lambda \mu \gamma_{t h}^{\alpha / 2}}{\left(\lambda^{2}-1\right) \bar{\gamma}^{\alpha / 2}},-\frac{1}{\lambda}\right) .
\end{aligned}
$$

Proof: The proof follows immediately by setting

$$
\eta=\frac{1-\lambda}{1+\lambda}
$$

in Corollary 5.

Remark 10: It readily follows from (124) that the CDF of the $\alpha-\lambda-\mu$ distribution can be expressed as,

$$
\begin{aligned}
F_{\gamma}(\gamma)= & \frac{(-1)^{2 \mu} \sqrt{\pi}(1-\lambda)^{\mu}(1+\lambda)^{\mu}}{\Gamma(\mu) 2^{\mu-\frac{1}{2}} \lambda^{2 \mu}} \\
& \times I e_{\mu+\frac{1}{2}+\frac{4(1-\alpha)}{\alpha^{2}}, \mu-\frac{1}{2}}\left(\frac{2 \lambda \mu \gamma^{\alpha / 2}}{\left(\lambda^{2}-1\right) \bar{\gamma}^{\alpha / 2}},-\frac{1}{\lambda}\right)
\end{aligned}
$$

whereas for the specific case that $\alpha=2$, equation (124) yields a closed-form expression for the OP over $\lambda-\mu$ fading channels, namely,

$$
\begin{aligned}
P_{\text {out }}= & \frac{(-1)^{2 \mu} \sqrt{\pi}(1-\lambda)^{\mu}(1+\lambda)^{\mu}}{\Gamma(\mu) 2^{\mu-\frac{1}{2}} \lambda^{2 \mu}} \\
& \times I e_{\mu-\frac{1}{2}, \mu-\frac{1}{2}}\left(\frac{2 \lambda \mu \gamma_{t h}}{\left(\lambda^{2}-1\right) \bar{\gamma}},-\frac{1}{\lambda}\right)
\end{aligned}
$$

which holds without restrictions on the value of $\lambda$ and $\mu$.

\section{Outage Probability Over $\alpha-\kappa-\mu$ Fading Channels}

The $\alpha-\kappa-\mu$ distribution was also proposed as a remarkably accurate model for accounting for small scale fading conditions. Its foundation is similar to that of $\alpha-\eta-\mu$ and $\alpha-\lambda-\mu$ distributions but it is differentiated in that it is complementary to these models while it characterizes efficiently line-of-sight (LOS) communication scenarios. This is explicitly illustrated in [87, Fig. 1] which demonstrates the whole range of modelling capabilities of the aforementioned non-linear fading models. The $\alpha-\kappa-\mu$ distribution includes as special cases the $\kappa-\mu, \alpha-\mu$, Rice, Nakagami- $m$ and Rayleigh distributions, while in terms of physical interpretation, $\kappa$ denotes the ratio between the in-phase dominant component and the quadrature dominant component, whereas $\alpha$ and $\mu$ parameters are defined as in $\alpha-\eta-\mu$ and $\alpha-\lambda-\mu$ distributions [87]. 
Definition 6: For $\alpha, \kappa, \mu, \rho \in \mathbb{R}^{+}$, the normalized envelope $P D F$ of the $\alpha-\kappa-\mu$ distribution is expressed as follows,

$$
p_{P}(\rho)=\frac{\alpha \mu(1+\kappa)^{\frac{\mu+1}{2}} I_{\mu-1}\left(2 \mu \sqrt{\kappa(1+\kappa)} \rho^{\alpha / 2}\right)}{\kappa^{\frac{\mu-1}{2}} e^{\kappa \mu} \rho^{1-\frac{\alpha(1+\mu)}{2}} e^{\mu(1+\kappa) \rho^{a}}} .
$$

Corollary 7: For $\alpha, \kappa, \mu, \bar{\gamma}, \gamma_{\text {th }} \in \mathbb{R}^{+}$, the OP over i.i.d $\alpha-\kappa-\mu$ fading channels can be expressed as follows,

$$
P_{\text {out }}=T \sqrt{\mu(1+\kappa) \gamma_{t h}^{\alpha / 2} / \bar{\gamma}^{\alpha / 2}}(2 \mu-1, \mu-1, \sqrt{\kappa \mu}) .
$$

Proof: Based on (126), the SNR PDF of the $\alpha-\kappa-\mu$ distribution is expressed as [87, eq. (6)],

$$
\begin{aligned}
p_{\gamma}(\gamma)= & \frac{\alpha \mu(1+\kappa)^{\frac{1+\mu}{2}}}{2 \kappa^{\frac{\mu-1}{2}} e^{\mu \kappa}} \frac{\gamma^{\frac{\alpha(1+\mu)}{4}}-1}{\bar{\gamma}^{\frac{\alpha(1+\mu)}{4}}} \\
& \times e^{-\mu(1+\kappa) \frac{\gamma^{\alpha / 2}}{\bar{\gamma}^{\alpha / 2}}} I_{\mu-1}\left(2 \mu \sqrt{\kappa(1+\kappa) \frac{\gamma^{a / 2}}{\bar{\gamma}^{\alpha / 2}}}\right) .
\end{aligned}
$$

Therefore, by substituting (128) into (115) it immediately follows that,

$$
\begin{aligned}
P_{\text {out }}= & \frac{\alpha \mu(1+\kappa)^{\frac{1+\mu}{2}}}{2 \kappa^{\frac{\mu-1}{2}} e^{\mu \kappa} \bar{\gamma}^{\frac{\alpha(1+\mu)}{4}}} \\
& \times \int_{0}^{\gamma_{t h}} \frac{\gamma^{\frac{\alpha(1+\mu)}{4}-1}}{e^{\mu(1+\kappa) \frac{\gamma^{\alpha / 2}}{\gamma^{\alpha / 2}}}} I_{\mu-1}\left(2 \mu \sqrt{\kappa(1+\kappa) \frac{\gamma^{a / 2}}{\bar{\gamma}^{\alpha / 2}}}\right) \mathrm{d} \gamma
\end{aligned}
$$

which upon performing a necessary change of variables and carrying out long but basic algebraic manipulations, it can be expressed as follows,

$$
P_{\text {out }}=\frac{2 e^{-\kappa \mu}}{(\kappa \mu)^{\frac{\mu-1}{2}}} \int_{0}^{\sqrt{\mu(1+\kappa) \gamma_{t h}^{\alpha / 2} / \bar{\gamma}^{\alpha / 2}}} \frac{\gamma^{\mu}}{e^{\gamma^{2}}} I_{\mu-1}(2 \sqrt{\kappa \mu} \gamma) \mathrm{d} \gamma .
$$

It is evident that (130) can be equivalently expressed as,

$$
\begin{aligned}
P_{\text {out }}= & 2(\sqrt{\mu \kappa})^{(\mu-1)-(2 \mu-1)+1} e^{-\mu \kappa} \\
& \times \int_{0}^{\sqrt{\mu(1+\kappa) \gamma_{t h}^{\alpha / 2} / \bar{\gamma}^{\alpha / 2}}} \frac{\gamma^{2 \mu-1}}{\gamma^{1-\mu} e^{\gamma^{2}}} I_{\mu-1}(2 \sqrt{\mu \kappa} \gamma) \mathrm{d} \gamma
\end{aligned}
$$

and thus, the above representation can be expressed in closedform in terms of the incomplete Toronto function. As a result, equation (127) is deduced, which completes the proof.

Remark 11: For the special case that $\alpha=2$, equation (127) reduces to the following closed-form expression for the $O P$ over $\kappa-\mu$ fading channels,

$$
P_{\text {out }}=T_{\sqrt{\mu(1+\kappa) \gamma_{\text {th }} / \bar{\gamma}}}(2 \mu-1, \mu-1, \sqrt{\kappa \mu}) .
$$

which to the best of the Authors knowledge, it has not been previously reported in the open technical literature.
D. Alternative Representations for the Outage Probability Over $\eta-\mu$ and $\lambda-\mu$ Fading Channels

The $\eta-\mu$ fading model has been used extensively in the analysis of conventional and emerging communication systems over generalized multipath fading channels. The corresponding OP was firstly addressed in [29], [33], and [64] for specific cases. In what follows, we derive exact closed-form expressions for the $\eta-\mu$ and $\lambda-\mu$ fading models which are valid for both integer and half-integer values of $\mu$.

Corollary 8: For $\eta, \mu, \bar{\gamma}, \gamma_{\text {th }} \in \mathbb{R}^{+}$, and $2 \mu \in \mathbb{N}$, the $O P$ over i.i.d $\eta-\mu$ fading channels can be expressed as follows,

$$
\begin{aligned}
P_{\text {out }}= & \frac{2 \sqrt{\pi} \eta^{\mu} \Gamma(2 \mu)_{2} F_{1}\left(\mu, \mu+\frac{1}{2}, \mu+\frac{1}{2}, \frac{(1-\eta)^{2}}{(1+\eta)^{2}}\right)}{\Gamma(\mu) \Gamma\left(\mu+\frac{1}{2}\right)(1+\eta)^{2 \mu}} \\
& -\sum_{l=0}^{2 \mu-1}\left(\begin{array}{c}
2 \mu-1 \\
l
\end{array}\right) \frac{\sqrt{\pi} l !(1+\eta)^{2 \mu-l-1} \gamma_{\text {th }}^{2 \mu-l-1}}{\bar{\gamma}^{2 \mu-l-1} \mu^{l-2 \mu} \eta^{\mu-l-1} \mu ! \Gamma\left(\mu+\frac{1}{2}\right)} \\
& \times \frac{\Phi_{1}\left(\mu, 1+l, 2 \mu ; 1-\eta, \frac{\mu\left(1-\eta^{2}\right) \gamma_{t h}}{\bar{\gamma} \eta}\right)}{2^{1-2 \mu} e^{-\frac{\mu(1+\eta) \gamma_{t h}}{\bar{\eta}}}}
\end{aligned}
$$

Proof: The proof follows with the aid of Theorem 8 and Corollary 5.

Corollary 9: For $\mu, \bar{\gamma}, \gamma_{\text {th }} \in \mathbb{R}^{+},-1<\lambda<1$ and $2 \mu \in \mathbb{N}$, the OP over i.i.d $\lambda-\mu$ fading channels can be expressed as,

$$
\begin{aligned}
P_{\text {out }}= & \frac{\sqrt{\pi}(1-\lambda)^{\mu}(1+\lambda)^{\mu} \Gamma(2 \mu)_{2} F_{1}\left(\mu, \mu+\frac{1}{2}, \mu+\frac{1}{2}, \lambda^{2}\right)}{\Gamma(\mu) \Gamma\left(\mu+\frac{1}{2}\right) 2^{2 \mu-1}} \\
& -\sum_{l=0}^{2 \mu-1}\left(\begin{array}{c}
2 \mu-1 \\
l
\end{array}\right) \frac{\sqrt{\pi} l ! \mu^{2 \mu-l} e^{-\frac{2 \mu \gamma_{t}}{\gamma(1-\lambda)}}}{(1+\lambda)^{\mu}(1-\lambda)^{\mu-l-1} m !} \\
& \times \frac{\Phi_{1}\left(\mu, 1+l, 2 \mu, \frac{2 \lambda}{1+\lambda}, \frac{4 \mu \lambda \gamma_{t h}}{\gamma(1+\lambda)(1-\lambda)}\right)}{\bar{\gamma}^{2 \mu-l-1} \gamma_{t h}^{1+l-2 \mu} \Gamma\left(\mu+\frac{1}{2}\right) 2^{l}} .
\end{aligned}
$$

Proof: The proof follows immediately by setting

$$
\eta=\frac{1-\lambda}{1+\lambda}
$$

in Corollary 8.

\section{E. Truncated Channel Inversion With Fixed Rate}

Transmission Over Rician Fading Channels

Corollary 10: For $n, \gamma_{0}, \gamma_{t h}, \bar{\gamma}, B \in \mathbb{R}^{+}$, the spectral efficiency for truncated channel inversion with fixed-rate (TIFR) policy over i.i.d. Rician fading channels can be expressed as follows,

$$
\begin{aligned}
\frac{C_{\mathrm{TIFR}}}{B}= & \log _{2}\left(1+\frac{\bar{\gamma}}{2\left(1+n^{2}\right) Q_{-1,0}\left(n \sqrt{2}, \sqrt{\frac{2 \gamma_{0}\left(1+n^{2}\right)}{\bar{\gamma}}}\right)}\right) \\
& \times\left\{1-T \sqrt{\left(1+n^{2}\right) \gamma_{t h} / \bar{\gamma}}(1,0, n)\right\}
\end{aligned}
$$

where $n$ denotes the Nakagami-n parameter, B is the corresponding channel bandwidth and $\gamma_{0}$ is the optimum cut-off SNR below which data transmission is suspended [10], [92].

Proof: It is widely known that Rice distribution has been traditionally used for accounting for multipath fading in LOS 
communication scenarios. The corresponding SNR per symbol follows the non-central chi-square distribution with its PDF given by [23, eq. (2.16)],

$$
p_{\gamma}(\gamma)=\frac{1+n^{2}}{\bar{\gamma} e^{n^{2}}} e^{-\left(1+n^{2}\right) \frac{\gamma}{\gamma}} I_{0}\left(2 n \sqrt{\frac{\left(1+n^{2}\right) \gamma}{\bar{\gamma}}}\right)
$$

where $n$ is related to the Ricean $K$ factor by $K=n^{2}$ and physically denotes the ratio of the LOS component to the average power of the scattered component [10]. It is also known that the inversion of the channel fading technique is based on adapting the transmitter power in order to maintain a constant SNR at the receiver. This technique often suffers a capacity penalty which can be combated by inverting the channel fading only above a pre-determined fixed cut-off fade depth $\gamma_{0}$ [10]. Mathematically, the C-TIFR is given by [10, eq. (15.36)], namely,

$$
C_{\mathrm{TIFR}}=B \log _{2}\left(1+\frac{1}{\int_{\gamma_{0}}^{\infty} \frac{p_{\gamma}(\gamma)}{\gamma} d \gamma}\right)\left\{1-P_{\text {out }}\right\} .
$$

As a result, in the case of Rician fading one obtains straightforwardly,

$$
\int_{\gamma_{0}}^{\infty} \frac{p_{\gamma}(\gamma)}{\gamma} \mathrm{d} \gamma=\frac{c}{e^{n^{2}}} \int_{\gamma_{0}}^{\infty} \frac{1}{\gamma} e^{-c \gamma} I_{0}(2 n \sqrt{c \gamma}) \mathrm{d} \gamma
$$

where

$$
c=\frac{\left(1+n^{2}\right)}{\bar{\gamma}} .
$$

Setting $y=\sqrt{2 a x}$ and thus, $x=y^{2} / 2 a$ and $\mathrm{d} y / \mathrm{d} x=\sqrt{a / 2 x}$ and after some basic algebraic manipulations it follows that,

$$
\begin{aligned}
\frac{c}{e^{n^{2}}} \int_{\gamma_{0}}^{\infty} \frac{1}{\gamma} e^{-c \gamma} I_{0}(2 n \sqrt{c \gamma}) \mathrm{d} \gamma \\
\quad=2 \frac{1+n^{2}}{\bar{\gamma}} \int_{\sqrt{\frac{2\left(1+n^{2}\right) \gamma_{0}}{\bar{\gamma}}}}^{\infty} \frac{e^{-\frac{\gamma^{2}+2 n^{2}}{2}}}{\gamma} I_{0}(\sqrt{2} n \gamma) \mathrm{d} \gamma .
\end{aligned}
$$

The above integral can be expressed in terms of the Nuttall $Q$-function. Furthermore, it is recalled that the Rice distribution constitutes a special case of the $\kappa-\mu$ distribution and thus, the corresponding OP can be readily deduced with the aid of (132) yielding,

$$
P_{\text {out }}=T \sqrt{\left(1+n^{2}\right) \gamma_{t h} / \bar{\gamma}}(1,0, n) .
$$

As a result, by substituting (141) and (142) in (138) yields (136), which completes the proof.

The optimum cut-off fade depth below which the data transmission is suspended is given by [10, eq. (15.5)], namely,

$$
\underbrace{\int_{\gamma_{0}}^{\infty} \frac{p_{\gamma}(\gamma)}{\gamma_{0}} \mathrm{~d} \gamma}_{\mathcal{I}_{12}}-\underbrace{\int_{\gamma_{0}}^{\infty} \frac{p_{\gamma}(\gamma)}{\gamma} \mathrm{d} \gamma}_{\mathcal{I}_{11}} \triangleq 1 .
$$

For the case of Rician fading we substitute (137) in (143) and by recalling that $\mathcal{I}_{11}$ can be expressed in closed-form according to (141) it follows that,

$$
\begin{gathered}
\underbrace{\frac{1+n^{2}}{\bar{\gamma} e^{n^{2}}} \int_{\gamma_{0}}^{\infty} e^{-\frac{1+n^{2}}{\bar{\gamma}} \gamma} I_{0}\left(2 n \sqrt{\frac{1+n^{2}}{\bar{\gamma}} \gamma}\right) \mathrm{d} \gamma}_{\mathcal{I}_{12}} \\
-2\left(1+n^{2}\right) Q_{-1,0}\left(n \sqrt{2}, \sqrt{\frac{2 \gamma_{0}\left(1+n^{2}\right)}{\bar{\gamma}}}\right)=1 .
\end{gathered}
$$

By setting

$$
y=\sqrt{\frac{2\left(1+n^{2}\right) \gamma}{\bar{\gamma}}}
$$

and thus,

$$
\gamma=\frac{\bar{\gamma} y^{2}}{2\left(1+n^{2}\right)}
$$

and

$$
\frac{\mathrm{d} y}{\mathrm{~d} \gamma}=\sqrt{\frac{1+n^{2}}{2 \gamma \bar{\gamma}}}
$$

the $\mathcal{I}_{12}$ term can be expressed in closed-form in terms of the Marcum $Q-$ function, namely,

$$
\mathcal{I}_{12}=Q_{1}\left(n \sqrt{2}, \sqrt{\frac{2 \gamma_{0}\left(1+n^{2}\right)}{\bar{\gamma}}}\right) .
$$

Therefore, by substituting (148) in (144) and after performing basic algebraic manipulations, the optimum cut-off SNR can be finally expressed as follows,

$$
\gamma_{0}=\frac{\bar{\gamma} Q_{1}\left(n \sqrt{2}, \sqrt{\frac{2 \gamma_{0}\left(1+n^{2}\right)}{\bar{\gamma}}}\right)}{\bar{\gamma}+2\left(1+n^{2}\right) Q_{-1,0}\left(n \sqrt{2}, \sqrt{\frac{2 \gamma_{0}\left(1+n^{2}\right)}{\bar{\gamma}}}\right)} .
$$

The above expression can be used in determining $\gamma_{0}$ numerically with the aid of popular software packages such as MATLAB and MATHEMATICA.

\section{F. Truncated Channel Inversion With Fixed Rate Transmission in MIMO Systems Over Rician Fading Channels}

In multiple-input multiple-output spatial multiplexing communications, truncated channel inversion with fixed rate can be applied to each eigen-mode in order to transform the fading eigen-modes into a set of parallel AWGN channels with the same average SNR [92]. This is expressed as

$$
\frac{1}{m \int_{\gamma_{0}}^{\infty} \frac{p_{\gamma}(\gamma)}{\gamma} \mathrm{d} \gamma}
$$

where, $p_{\gamma}(\gamma)$ is the PDF of the corresponding fading statistics and $m$ are the non-zero positive real eigenvalues of the noncentral Wishart-type random matrix $\mathbf{H} \mathbf{H}^{\mathcal{H}}$, with $\mathcal{H}$ denoting the Hermitian operator. Also, $\gamma_{0}$ is the predetermined SNR threshold which is selected accordingly for either guaranteeing 


$$
C_{\mathrm{em}-\mathrm{tifr}}^{1, n}=\log _{2}\left(1+\frac{\bar{\gamma}\left(2 K \mathbf{m}^{\mathcal{H}} \mathbf{m}\right)^{\frac{n-1}{2}}}{2(K+1) Q_{n-2, n-1}\left(\sqrt{2 K \mathbf{m}^{\mathcal{H}} \mathbf{m}}, \sqrt{\frac{2(K+1) \gamma_{0}}{\bar{\gamma}}}\right)}\right)\left\{1-T \sqrt{\frac{(K+1) \gamma_{0}}{\bar{\gamma}}}\left(2 n-1, n-1, \sqrt{K \mathbf{m}^{\mathcal{H}} \mathbf{m}}\right)\right\}
$$

a required OP or for maximizing the achievable fixed transmission rate of the eigen-mode truncated channel inversion (em-ti) policy with capacity:

$$
C_{\mathrm{em}-\mathrm{tifr}}^{m, n}=m \log _{2}\left(1+\frac{1}{m \int_{\gamma_{0}}^{\infty} \frac{p_{\gamma}(\gamma)}{\gamma} \mathrm{d} \gamma}\right) \int_{\gamma_{0}}^{\infty} p_{\gamma}(\gamma) \mathrm{d} \gamma
$$

where $n$ are non-zero mean circularly symmetric Gaussian random variables whose sum denote the non-zero eigenvalue $\lambda$ [93], [95].

Corollary 11: For $K, n, \gamma_{0}, \bar{\gamma} \in \mathbb{R}^{+}$and $\gamma_{t h} \in \mathbb{R}^{+}$, the em-tifr capacity of MISO/SIMO communication systems over uncorrelated Rician fading channels can be expressed according to (151), as shown at the top of the page, with $K$ denoting the Rician $K$-factor and $\boldsymbol{m}$ being the $N$-dimensional deterministic vector that accounts for the corresponding LOS component.

Proof: With the aid of the SNR PDF for uncorrelated Rician fading channels in [95, eq. (29)] and recalling that

$$
\int_{\gamma_{0}}^{\infty} p_{\gamma}(\gamma) \mathrm{d} \gamma=1-P_{\text {out }}
$$

it immediately follows that,

$$
\begin{aligned}
P_{\text {out }}^{1, n}= & \frac{(K+1)^{\frac{n+1}{2}} e^{-K \mathbf{m}^{\mathcal{H}} \mathbf{m}}}{\bar{\gamma}^{\frac{n+1}{2}}\left(K \mathbf{m}^{\mathcal{H}} \mathbf{m}\right)^{\frac{n-1}{2}}} \\
& \times \int_{0}^{\gamma_{0}} \frac{\gamma^{\frac{n-1}{2}}}{e^{\frac{(K+1) \gamma}{2}}} I_{n-1}\left(2 \sqrt{\frac{(K+1) K \mathbf{m}^{\mathcal{H}} \mathbf{m} \gamma}{\bar{\gamma}}}\right) \mathrm{d} \gamma .
\end{aligned}
$$

The above representation can be expressed in terms of the incomplete Toronto function, namely,

$$
P_{\text {out }}^{1, n}=T \sqrt{\frac{(K+1) \gamma_{0}}{\bar{\gamma}}}\left(2 n-1, n-1, \sqrt{K \mathbf{m}^{\mathcal{H}} \mathbf{m}}\right) .
$$

By substituting (154) in [95, eq. (38)], one obtains (151), which completes the proof.

The optimal cutoff threshold for (151) has to satisfy [95, eq. (34)], namely,

$$
\begin{aligned}
\gamma_{0}= & Q_{n}\left(\sqrt{2 K \mathbf{m}^{\mathcal{H}} \mathbf{m}}, \sqrt{2 \mu_{K} \gamma_{0}}\right) \\
& -\frac{2^{\frac{3-n}{2}} \mu_{K} \gamma_{0}}{\left(K \mathbf{m}^{\mathcal{H}} \mathbf{m}\right)^{\frac{n-1}{2}}} Q_{n-2, n-1}\left(\sqrt{2 K \mathbf{m}^{\mathcal{H}} \mathbf{m}}, \sqrt{2 \mu_{K} \gamma_{0}}\right)
\end{aligned}
$$

where

$$
\mu_{K}=\frac{K+1}{\bar{\gamma}}
$$

The above expression can be further elaborated and an exact closed-form expression for $\gamma_{0}$ can be deduced.

Lemma 9: For $K, \bar{\gamma}, n \in \mathbb{R}^{+}$and with $Q_{m, n}^{-1}(a, b)$ denoting the inverse Nuttall $Q$-function, the following closed-form expression holds for the optimal cut-off threshold in (151),

$$
\gamma_{0}=\frac{\left[Q_{n-2, n-1}^{-1}\left(\sqrt{2 K \boldsymbol{m}^{\mathcal{H}} \boldsymbol{m}},-\frac{\left(2 K \boldsymbol{m}^{\mathcal{H}} \boldsymbol{m}\right)^{\frac{n-1}{2}}}{\mu_{K}}\right)\right]^{2}}{2 \mu_{K}} .
$$

Proof: By taking the first derivative of (155) w.r.t. $\gamma_{0}$ it immediately follows that,

$$
\begin{gathered}
\frac{\partial Q_{n}\left(\mathcal{A}, \sqrt{2 \mu_{K} \gamma_{0}}\right)}{\partial \gamma_{0}}-\frac{2 \mu_{K} \gamma_{0}}{\mathcal{A}^{n-1}} \frac{\partial Q_{n-2, n-1}\left(\mathcal{A}, \sqrt{2 \mu_{K} \gamma_{0}}\right)}{\partial \gamma_{0}} \\
-\frac{2 \mu_{K}}{\mathcal{A}^{n-1}} Q_{n-2, n-1}\left(\mathcal{A}, \sqrt{2 \mu_{K} \gamma_{0}}\right)=1
\end{gathered}
$$

where

$$
\mathcal{A}=\sqrt{2 K \mathbf{m}^{\mathcal{H}} \mathbf{m}} .
$$

After performing the above derivatives it follows that,

$$
\begin{aligned}
\mathcal{A}^{1-n} \frac{2 \mu_{K} \gamma_{0} I_{n-1}\left(\mathcal{A} \sqrt{\mu_{K} \gamma_{0}}\right)}{\left(2 \mu_{K} \gamma_{0}\right)^{1-\frac{n}{2}} e^{\frac{2 \mu_{K} \gamma_{0}+\mathcal{A}^{2}}{2}}} & -\frac{2 \mu_{K}}{\mathcal{A}^{n-1}} Q_{n-2, n-1}\left(\mathcal{A}, \sqrt{2 \mu_{K} \gamma_{0}}\right) \\
- & \frac{\left(2 \mu_{K} \gamma_{0}\right)^{\frac{n}{2}} I_{n-1}\left(\mathcal{A} \sqrt{\mu_{K} \gamma_{0}}\right)}{\mathcal{A}^{n-1} e^{\frac{2 \mu_{K} \gamma_{0}+\mathcal{A}^{2}}{2}}}=1
\end{aligned}
$$

which after some basic algebraic manipulations becomes,

$$
Q_{n-2, n-1}\left(\mathcal{A}, \sqrt{2 \mu_{K} \gamma_{0}}\right)=-\frac{\mathcal{A}^{n-1}}{\mu_{K}} .
$$

With the aid of the inverse Nuttall $Q$-function, it immediately follows that,

$$
\sqrt{2 \mu_{K} \gamma_{0}}=Q_{n-2, n-1}^{-1}\left(\sqrt{2 K \mathbf{m}^{\mathcal{H}} \mathbf{m}},-\frac{\mathcal{A}^{n-1}}{\mu_{K}}\right) .
$$

As a result, by solving w.r.t $\gamma_{0}$ one obtains (157) which completes the proof.

In MIMO communication scenarios over uncorrelated Rician fading channels, the random matrix $\mathbf{H} \mathbf{H}^{\mathcal{H}}$ follows a Wishart type distribution. The corresponding PDF of a single unordered eigenvalue $\lambda$ was given in [96, Corollary 1], and then in [95, eq. (69)], which with the aid of $\gamma=\lambda \bar{\gamma}$ it is expressed as,

$$
\begin{aligned}
p_{\gamma}(\gamma)= & \frac{K_{m, n}^{\omega_{t}}}{m} \sum_{i=1}^{m} \sum_{j=1}^{m-t} \frac{c_{i j}^{(t)} \gamma^{d+i+j-2}}{\mu_{K}^{1-d-i-j} e^{\mu_{K} \gamma}} \\
& +K_{m, n}^{\omega_{t}} \sum_{i=1}^{m} \sum_{j=m-t+1}^{m} \frac{c_{i j}^{(t)} \mu_{K}^{d+i}{ }_{0} F_{1}\left(d+1 ; \mu_{K} \gamma \omega_{j}\right)}{m d ! \gamma^{1-d-i} e^{\mu_{K} \gamma}}
\end{aligned}
$$


where $d=n-m$ whereas $c_{i j}^{(t)}$ and $K_{m, n}^{\omega_{t}}$ are given by [95, eq. (69)] and [95, eq. (71)], respectively. Also, $\omega_{m-t+1}, \ldots, \omega_{m}$ are $t$ distinct eigenvalues of the noncentrality parameter of the distribution that can be represented in the form of the following as a column vector,

$$
\omega_{t}=\left[\omega_{m-t+1}, \ldots, \omega_{m}\right]^{T} .
$$

To this effect, the overall capacity of the em-ti transmission policy in MIMO systems is given by [95, eq. (47)], namely,

$$
C_{\mathrm{em}-\mathrm{ti}}^{m, n}=m \log _{2}\left(1+\kappa_{\mathrm{em}-\mathrm{ti}}^{m, n}\left(\gamma_{0}\right)\right) \times\left(1-P_{\mathrm{out}}^{\mathrm{em}}\right)
$$

where $\kappa_{\mathrm{em}-\mathrm{ti}}^{m, n}\left(\gamma_{0}\right), P_{\mathrm{out}}^{\mathrm{em}}$ are given in (166) and (167), respectively, along with the optimal cut-off $\gamma_{0}$ in [95, eq. (44)] which is given in (168), as shown at the bottom of the page. Evidently, the performance measures in (166)-(168) can be computed accurately and straightforwardly with the aid of the proposed expressions for the $Q_{m, n}(a, b)$ function in Sec. II.

\section{CONCLUSIONS}

New analytic expressions were derived for a set of important special functions in wireless communication theory, namely, the Nuttall $Q$-function, the incomplete Toronto function, the Rice $I e-$ function and the incomplete Lipschitz Hankel integrals. These expressions include closed-form expressions for general and specific cases as well as tight upper and lower bounds, polynomial representations and approximations. Explicit relationships in terms of these functions were also provided for specific cases of the Humbert $\Phi_{1}$ and Kampé de Fériet special functions. The derived expressions are rather useful both analytically and computationally because although the considered functions have been used widely in analyses relating to wireless communications, they are neither tabulated nor built-in functions in popular mathematical software packages such as MATLAB, MATHEMATICA and MAPLE. As an example, the offered results were indicatively employed in deriving novel analytic expressions for the outage probability over $\alpha-\eta-\mu, \alpha-\lambda-\mu$ and $\alpha-\kappa-\mu$ fading channels as well as for the truncated capacity with channel inversion in single-antenna and multi-antenna communications under Rician multipath fading conditions.

\section{APPENDIX A}

PROOF OF THEOREM 1

Equation (1) can be alternatively written as,

$$
\begin{aligned}
Q_{m, n}(a, b)= & \underbrace{e^{-\frac{a^{2}}{2}} \int_{0}^{\infty} x^{m} e^{-\frac{x^{2}}{2}} I_{n}(a x) \mathrm{d} x}_{\mathcal{G}} \\
& -e^{-\frac{a^{2}}{2}} \int_{0}^{b} x^{m} e^{-\frac{x^{2}}{2}} I_{n}(a x) \mathrm{d} x .
\end{aligned}
$$

By utilizing [63, eq. (8.406.3)] and [63, eq. (6.621.1)] in $\mathcal{G}$ it follows that,

$$
\mathcal{G}=\frac{a^{n} \Gamma\left(\frac{m+n+1}{2}\right){ }_{1} F_{1}\left(\frac{m+n+1}{2}, 1+n, \frac{a^{2}}{2}\right)}{n ! e^{\frac{a^{2}}{2}} 2^{\frac{n-m+1}{2}}} .
$$

Substituting (170) in (169) and expanding the $I_{n}(x)$ function according to $[63$, eq. (8.445)] one obtains,

$$
\begin{aligned}
Q_{m, n}(a, b)= & \mathcal{G} \\
& \times \sum_{l=0}^{\infty} \frac{a^{n+2 l} e^{-\frac{a^{2}}{2}}}{l ! \Gamma(n+l+1) 2^{n+2 l}} \\
& \times \int_{0}^{b} x^{m+n+2 l} e^{-\frac{x^{2}}{2}} \mathrm{~d} x .
\end{aligned}
$$

Both $I_{n}(x)$ and $\exp (x)$ are entire functions and the limits of (171) are finite. Thus, substituting [63, eq. (1.211.1)] in (171), the resulting integral can be straightforwardly evaluated analytically yielding (172), as shown at the bottom of the page. To this effect and using the Pochhammer symbol

$$
(a)_{n}=\frac{\Gamma(a+n)}{\Gamma(a)}
$$

$$
\begin{aligned}
& \kappa_{\mathrm{em}-\mathrm{ti}}^{m, n}\left(\gamma_{0}\right)=\frac{\mu_{K} / K_{m, n}^{\omega_{t}}}{\sum_{i=1}^{m}\left[\sum_{j=1}^{m-t} c_{i j}^{(t)} \Gamma\left(d+i+j-2, \mu_{K} \gamma_{0}\right)+\sum_{j=m-t+1}^{m} c_{i j}^{(t)} \frac{Q_{d+2 i-3, d}\left(\sqrt{2 \omega_{j}}, \sqrt{2 \mu_{K} \gamma_{0}}\right)}{\left.2^{i-2+\frac{d}{2} \omega_{j}^{d / 2} e^{-\omega_{t}}}\right]}\right.} \\
& P_{\mathrm{out}}^{\mathrm{em}}=1-\sum_{i=1}^{m}\left[\sum_{j=1}^{m-t} \frac{\Gamma\left(d+i+j-2, \mu_{K} \gamma_{0}\right)}{m\left[K_{m, n}^{\omega_{t}} c_{i j}^{(t)}\right]^{-1}}+\sum_{j=m-t+1}^{m} \frac{Q_{d+2 i-3, d}\left(\sqrt{2 \omega_{j}}, \sqrt{2 \mu_{K} \gamma_{0}}\right) e^{\omega_{t}}}{m\left[K_{m, n}^{\omega_{t}} c_{i j}^{(t)}\right]^{-1} 2^{i-2+\frac{d}{2}} \omega_{j}^{d / 2}}\right] \\
& \gamma_{0}=\sum_{i=1}^{m} \sum_{j=1}^{m-t} K_{m, n}^{\omega_{t}} c_{i j}^{(t)}\left[\Gamma\left(d+i+j-1, \mu_{K} \gamma_{0}\right)-\mu_{K} \gamma_{0} \Gamma\left(d+i+j-1, \mu_{K} \gamma_{0}\right)\right] \\
& +\sum_{i=1}^{m} \sum_{j=m-t+1}^{m} \frac{Q_{d+2 i-1, d}\left(\sqrt{2 \omega_{j}}, \sqrt{2 \mu_{K} \gamma_{0}}\right)-\sqrt{2 \mu_{K} \gamma_{0}} Q_{d+2 i-2, d}\left(\sqrt{2 \omega_{j}}, \sqrt{2 \mu_{K} \gamma_{0}}\right)}{\left[K_{m, n}^{\omega_{t}} c_{i j}^{(t)}\right]^{-1} 2^{i-1+\frac{d}{2}} \omega_{j}^{d / 2} e^{-\omega_{j}}} . \\
& Q_{m, n}(a, b)=\mathcal{G}-\sum_{l=0}^{\infty} \sum_{i=0}^{\infty} \frac{(-1)^{i} a^{n+2 l} b^{m+2 l+2 i+n+1} e^{-\frac{a^{2}}{2}}}{l ! i ! \Gamma(n+l+1) 2^{n+i+2 l}(m+2 l+2 i+n+1)}
\end{aligned}
$$




$$
\begin{aligned}
& Q_{m, n}(a, b)=\mathcal{G}-\frac{a^{n} b^{n}}{2^{n} e^{\frac{a^{2}}{2}}} \sum_{l=0}^{\infty} \sum_{i=0}^{\infty} \frac{(-1)^{i} a^{2 l} b^{m+2 l+2 i+1}(m+2 i+n+1)_{2 l}(m+n+1)_{2 i}(m+n) !}{l ! i !(n+l) ! 2^{i+2 l}(m+2 i+n+2)_{2 l}(m+n+2)_{2 i} \Gamma(m+n+2)} . \\
& Q_{m, n}(a, b)=\mathcal{G}-\frac{a^{n} e^{-\frac{a^{2}}{2}}}{m+n+1} \sum_{l=0}^{\infty} \sum_{i=0}^{\infty} \frac{(-1)^{i} a^{2 l} b^{m+n+2 l+2 i+1}\left(\frac{m+n+1}{2}+i\right)_{l}\left(\frac{m+n+1}{2}\right)_{i}}{l ! i !(n+1)_{l}\left(\frac{m+n+1}{2}+1+i\right)_{l}\left(\frac{m+n+1}{2}+1\right)_{i} 2^{n+i+2 l}} . \\
& Q_{m, n}(a, b)=\mathcal{G}-\frac{e^{-\frac{a^{2}}{2}} a^{n} b^{m+n+1}}{(m+n+1) n ! 2^{n}} \sum_{l=0}^{\infty} \sum_{i=0}^{\infty} \frac{\left(\frac{m+n+1}{2}\right)_{l+i}}{(n+1)_{l}\left(\frac{m+n+3}{2}\right)_{l+i}} \frac{\left(\frac{a^{2} b^{2}}{4}\right)^{l}}{l !} \frac{\left(-\frac{b^{2}}{2}\right)^{i}}{i !} .
\end{aligned}
$$

while recalling that

$$
\begin{aligned}
a & =\frac{a !}{(a-1) !} \\
& =\frac{\Gamma(a+1)}{\Gamma(a)}
\end{aligned}
$$

one obtains (176), as shown at the top of the page. Which upon using the identity,

$$
(2 x)_{2 n}=2^{2 n}(x)_{n}\left(x+\frac{1}{2}\right)_{n}
$$

it leads to (178), as shown at the top of the page. By subsequently expressing each term of the form $(a+m)_{n}$ as follows,

$$
(a+m)_{n}=\frac{\Gamma(a+m+n)}{\Gamma(a+m)}=\frac{(a)_{m+n}}{(a)_{m}}
$$

and performing some basic algebraic manipulations (178) can be re-written according to (180), as shown at the top of the page.

Importantly, this double series representation can be expressed in terms of the KdF function [78], [82]. Therefore, by performing the necessary change of variables and substituting in (180), eq. (7) is deduced thus, completing the proof.

\section{APPENDIX B \\ PROOF OF PROPOSITION 1}

Tight polynomial approximations for the modified Bessel function of the first kind were derived by Gross et al in [83]. Based on this, by substituting [83, eq. (19)] in (1) one obtains,

$$
Q_{m, n}(a, b) \simeq \sum_{l=0}^{p} \frac{\Gamma(p+l) p^{1-2 l} a^{n+2 l}}{l !(p-l) !(n+l) !} \int_{b}^{\infty} \frac{x^{2 l+m+n}}{2^{n+2 l} e^{\frac{x^{2}+a^{2}}{2}}} \mathrm{~d} x .
$$

By recalling that,

$$
\Gamma(x) \triangleq(x-1) !
$$

which holds for when $x \in \mathbb{R}^{+}$, and expressing the above integral according to [63, eq. (8.350.2)] yields (8). To this effect and for the specific case that $\frac{m+n+1}{2} \in \mathbb{N}$, the $\Gamma(a, x)$ function can be expressed in terms of a finite series according to [63, eq. (8.352.4)]. Therefore, by performing the necessary change of variables and substituting in (8) yields (9), which completes the proof.

\section{APPENDIX C \\ PROOF OF THEOREM 2}

By expressing the $I_{n}(x)$ function according to [63, eq. (8.467)] and substituting in (35) yields,

$$
\begin{aligned}
T_{B}(m, n, r)= & \sum_{k=0}^{n-\frac{1}{2}} \frac{r^{n-m-k+\frac{1}{2}}\left(n+k-\frac{1}{2}\right) !}{k ! \sqrt{\pi}\left(n-k-\frac{1}{2}\right) ! 2^{2 k} e^{r^{2}}} \\
& \times\left\{\int_{0}^{B}(-1)^{k} t^{m-n-k-\frac{1}{2}} e^{-t^{2}} e^{2 r t} \mathrm{~d} t\right. \\
& \left.+\int_{0}^{B}(-1)^{n+\frac{1}{2}} t^{m-n-k-\frac{1}{2}} e^{-t^{2}} e^{-2 r t} \mathrm{~d} t\right\}
\end{aligned}
$$

which can be equivalently expressed as follows,

$$
\begin{aligned}
T_{B}(m, n, r)= & \sum_{k=0}^{n-\frac{1}{2}} \frac{\left(n+k-\frac{1}{2}\right) ! r^{n-m-k+\frac{1}{2}}}{\sqrt{\pi} k !\left(n-k-\frac{1}{2}\right) ! 2^{2 k}} \\
& \times\{\underbrace{\int_{0}^{B}(-1)^{n+\frac{1}{2}} t^{L-k} e^{-(t+r)^{2}} \mathrm{~d} t}_{\mathcal{I}_{3}} \\
& +\underbrace{\int_{0}^{B}(-1)^{k} t^{L-k} e^{-(t-r)^{2}} \mathrm{~d} t}_{\mathcal{I}_{4}}\}
\end{aligned}
$$

where $L=m-n-\frac{1}{2}$. The $\mathcal{I}_{3}$ and $\mathcal{I}_{4}$ can be also expressed in terms of [82, eq. (1.3.3.18)] which yields (185), as shown at the bottom of the page. Importantly, the $\mathcal{I}_{5}$ and $\mathcal{I}_{6}$ integrals

$$
T_{B}(m, n, r)=\sum_{k=0}^{n-\frac{1}{2}} \sum_{l=0}^{L} \frac{r^{-(2 k+l)}\left(n+k-\frac{1}{2}\right) !(L-k) !}{\sqrt{\pi} k ! l !\left(n-k-\frac{1}{2}\right) !(L-k-l) !}\{(-1)^{m-l} \underbrace{\int_{0}^{B+r} \frac{t^{l} e^{-t^{2}}}{2^{2 k}} \mathrm{~d} t}_{\mathcal{I}_{5}}+(-1)^{k} \underbrace{\int_{0}^{B-r} \frac{t^{l} e^{-t^{2}}}{2^{2 k}} \mathrm{~d} t}_{\mathcal{I}_{6}}\} .
$$




$$
\begin{aligned}
& T_{B}(m, n, r)=2 r^{n-m+1} e^{-r^{2}} \sum_{l=0}^{\infty} \sum_{i=0}^{\infty} \frac{(-1)^{i} r^{n+2 l} B^{m+2 l+2 i+1}(m+2 i+1)_{2 l}(m+1)_{2 i} \Gamma(m+1)}{l ! i !(n+1)_{l} \Gamma(n+1)(m+2 i+2)_{2 l}(m+2)_{2 i} \Gamma(m+2)} . \\
& T_{B}(m, n, r)=\frac{2 r^{n-m+1} e^{-r^{2}}}{(m+1)} \sum_{l=0}^{\infty} \sum_{i=0}^{\infty} \frac{(-1)^{i} r^{n+2 l} B^{m+2 l+2 i+1}\left(\frac{m+1}{2}+i\right)_{l}\left(\frac{m+1}{2}\right)_{i}}{l ! i ! \Gamma(n+l+1)\left(\frac{m+1}{2}+1+i\right)_{l}\left(\frac{m+1}{2}+1\right)_{i}} .
\end{aligned}
$$

can be expressed in terms of the $\gamma(a, x)$ function. Hence, by making the necessary change of variables and substituting in (185) yields (38), which completes the proof.

\section{APPENDIX D}

\section{PROOF OF LEMMA 2}

By performing a change of variables and the integral limits in $T_{B}(m, n, r)$ and utilizing [63, eq. (8.406.3)] and [63, eq. (6.631.1)] one obtains,

$$
\begin{aligned}
T_{B}(m, n, r)= & \frac{\Gamma\left(\frac{m+1}{2}\right){ }_{1} F_{1}\left(\frac{m+1}{2}, 1+n, r^{2}\right)}{r^{m-2 n-1} \Gamma(n+1) e^{r^{2}}} \\
& -\frac{2 e^{-r^{2}}}{r^{m-n-1}} \int_{B}^{\infty} t^{m-n} e^{-t^{2}} I_{n}(2 r t) \mathrm{d} t .
\end{aligned}
$$

By setting $u=\sqrt{2} t$ and performing long but basic algebraic representations, the above integral can be expressed in closedform in terms of the Nuttall $Q$-function which yields (39). This completes the proof.

\section{APPENDIX E}

\section{PROOF OF THEOREM 4}

The $\exp (x)$ and $I_{n}(x)$ functions in (35) are entire and can be expanded since the integration interval is finite. To this end, by making the necessary variable transformation in [63, eq. (1.211.1)] and [63, eq. (8.445)], respectively, and substituting in (35) it follows that,

$$
\begin{aligned}
T_{B}(m, n, r)= & 2 r^{n-m+1} e^{-r^{2}} \\
& \times \sum_{l=0}^{\infty} \sum_{i=0}^{\infty} \frac{(-1)^{i} r^{n+2 l} B^{m+2 l+2 i+1}}{l ! i ! \Gamma(n+l+1)(m+2 l+2 i+1)} .
\end{aligned}
$$

By recalling that

$$
\begin{aligned}
(x+y) & =\frac{(x+y) !}{(x+y-1) !} \\
& =\frac{(x+y) !}{\Gamma(x+y)}
\end{aligned}
$$

and that

$$
\Gamma(x+n)=(x)_{n} \Gamma(x)
$$

and subsequently substitute in (187) yields (191), as shown at the top of the page. With the aid of the identity,

$$
(2 x)_{2 n}=2^{2 n}(x)_{n}\left(x+\frac{1}{2}\right)_{n}
$$

and after some basic algebraic manipulations (191) can be alternatively expressed according to (193), as shown at the top of the page. Notably, each term of the form $(x+i)_{l}$ can be equivalently expressed as follows,

$$
\frac{\Gamma(x+i+l)}{\Gamma(x+i)}=\frac{(x)_{l+i}}{(x)_{i}} .
$$

To this effect, by substituting accordingly in (193) one obtains,

$$
\begin{aligned}
T_{B}(m, n, r)= & \frac{2 r^{2 n-m+1} B^{m+1}}{n !(m+1) e^{r^{2}}} \\
& \times \sum_{l=0}^{\infty} \sum_{i=0}^{\infty} \frac{(-1)^{i} r^{2 l} B^{2 l+2 i}\left(\frac{m+1}{2}\right)_{l+i}}{l ! i !(n+1)_{l}\left(\frac{m+1}{2}+1\right)_{l+i}} .
\end{aligned}
$$

The above series can be expressed in closed-form in terms of the KdF Function in [63], [78], and [82]. Therefore, by making the necessary change of variables and substituting in (195), one obtains (46), which completes the proof.

\section{APPENDIX F \\ PROOF OF THEOREM 5}

The $I_{n}(x)$ function is monotonically decreasing with respect to its order $n$. Therefore, for an arbitrary positive real quantity $a \in \mathbb{R}^{+}$, it can be claimed straightforwardly that $I_{n \pm a}(x) \lessgtr$ $I_{n}(x)$. By applying this identity in (59) the $I e(k, x)$ can be upper bounded as follows,

$$
I e(k, x)<1-e^{-x} I_{0}(k x)+k \int_{0}^{x} e^{-t} I_{\frac{1}{2}}(k t) \mathrm{d} t .
$$

With the aid of the closed-form expression in [63, eq. (8.467)] it follows that

$$
I_{\frac{1}{2}}(k t)=\frac{e^{k t}-e^{-k t}}{\sqrt{2 \pi k t}}
$$

By substituting this in (196) one obtains,

$$
I e(k, x)<1-e^{-x} I_{0}(k x)+k \underbrace{\int_{0}^{x} e^{-t}\left[\frac{e^{k t}-e^{-k t}}{\sqrt{2 \pi k t}}\right] \mathrm{d} t}_{\mathcal{I}_{8}} .
$$

The $\mathcal{I}_{8}$ integral can be expressed in closed-form in terms of the $\operatorname{erf}(x)$ function, namely,

$$
\mathcal{I}_{8}=\sqrt{\frac{k}{2}}\left[\frac{\operatorname{erf}(\sqrt{x} \sqrt{1-k})}{\sqrt{1-k}}-\frac{\operatorname{erf}(\sqrt{x} \sqrt{1+k})}{\sqrt{1+k}}\right] .
$$

By substituting (199) in (196), equation (65) is deduced. Likewise, based on the aforementioned monotonicity property of the $I_{n}(x)$ function, it is easily shown that $I_{\frac{3}{2}}(x)<I_{1}(x)$. To this effect and by performing the necessary change of variables and substituting in (59), one obtains the following inequality,

$$
I e(k, x)>1-e^{-x} I_{0}(k x)+k \int_{0}^{x} e^{-t} I_{\frac{3}{2}}(k t) \mathrm{d} t .
$$


It is noted that a similar inequality can be obtained by exploiting the monotonicity properties of the Marcum $Q$-function which is strictly increasing w.r.t $m$. Based on this it follows that $Q_{1}(a, b)>Q_{0.5}(a, b)$, which upon substituting in [62, eq. (2c)] yields,

$$
I e(k, x)>\frac{1}{\sqrt{1-k^{2}}}\left[2 Q_{\frac{1}{2}}(a, b)-e^{-x} I_{0}(k x)-1\right] .
$$

Importantly, according to [46, eq. (27)],

$$
Q_{0.5}(a, b)=Q(b+a)+Q(b-a) .
$$

Therefore, by substituting in (201) and applying the identity

$$
\operatorname{erf}(x)=1-2 Q(x \sqrt{2})
$$

equation (66) is deduced, which completes the proof.

\section{APPENDIX G \\ PROOF OF THEOREM 6}

By changing the integral limits in (57) and expressing the $I_{n}(x)$ function according to [63, eq. (9.238.2)] it follows that,

$$
I e(k, x)=\frac{1}{\sqrt{1-k^{2}}}-\int_{x}^{\infty} e^{-t(1+k)}{ }_{1} F_{1}\left(\frac{1}{2}, 1,2 k t\right) \mathrm{d} t
$$

where the

$$
\int_{0}^{\infty} \exp (-t) I_{0}(k t) \mathrm{d} t
$$

integral was expressed in closed-form with the aid of [63, eq. (8.406.3)] and [63, eq. (6.621.1)]. By subsequently setting

$$
u=2 k t-2 k x
$$

and therefore, $t=(u+2 k x) / 2 k$ and $\mathrm{d} u / \mathrm{d} t=2 k$, it follows that,

$$
\begin{aligned}
I e(k, x)= & \frac{1}{\sqrt{1-k^{2}}} \\
& -\frac{e^{-(1+k) x}}{2 k} \int_{0}^{\infty} e^{-\frac{(1+k)}{2 k}{ }_{1} F_{1}}\left(\frac{1}{2}, 1, u+2 k x\right) \mathrm{d} u .
\end{aligned}
$$

The above integral can be expressed in terms of the confluent Appell function or Humbert function $\Phi_{1}$ with the aid of [86, eq. (3.35.1.9)]. Based on this, by making the necessary variable transformation and substituting in (206) yields (69) thus, completing the proof.

\section{APPENDIX H \\ PROOF OF THEOREM 7}

By making the necessary change of variables in [63, eq. (8.467)] and substituting in (78) one obtains,

$$
\begin{aligned}
I e_{m, n}(x ; a)= & \sum_{k=0}^{n-\frac{1}{2}} \frac{\left(n+k-\frac{1}{2}\right) ! 2^{-k-\frac{1}{2}}}{\sqrt{\pi} k !\left(n-k-\frac{1}{2}\right) !} \\
& \times\left\{(-1)^{k} \int_{0}^{x} y^{\mathcal{P}} e^{y} e^{-a y} \mathrm{~d} y\right. \\
& \left.+(-1)^{n+\frac{1}{2}} \int_{0}^{x} x^{\mathcal{P}} e^{-y} e^{a y} \mathrm{~d} y\right\}
\end{aligned}
$$

where $\mathcal{P}=m-k+\frac{1}{2}$. Both integrals in (207) can be expressed in closed-form in terms of the $\gamma(a, x)$ function. Hence, after basic algebraic manipulations one obtains (80), which completes the proof.

\section{APPENDIX I \\ PROOF OF THEOREM 8}

The $I_{n}(x)$ based representation in (78) can be equivalently expressed as follows,

$$
I e_{m, n}(x ; a)=\int_{0}^{\infty} y^{m} e^{-a y} I_{n}(y) \mathrm{d} y-\int_{x}^{\infty} y^{m} e^{-a y} I_{n}(y) \mathrm{d} y .
$$

The first integral in (208) can be expressed in closed-form according to [63, eq. (8.406.3)] and [63, eq. (6.621.1)]. To this effect and by re-writing the second integral by applying [63, eq. (9.238.2)] one obtains,

$$
I e_{m, n}(x ; a)=\frac{(m+n) !_{2} F_{1}\left(\frac{m+n+1}{2}, \frac{m+n}{2}+1 ; 1+n ; \frac{1}{a^{2}}\right)}{2^{n} a^{m+n+1} n !}
$$

The above integral can be expressed as,

$$
\begin{aligned}
\int_{x}^{\infty} & \frac{y^{m+n}}{e^{y(1+a)}}{ }_{2} F_{1}\left(n+\frac{1}{2}, 1+2 n, 2 y\right) \mathrm{d} y \\
= & \int_{0}^{\infty} \frac{(y+x)^{m+n}{ }_{2} F_{1}\left(n+\frac{1}{2}, 1+2 n, 2(x+y)\right)}{e^{y(1+a)} e^{x(1+a)}} \mathrm{d} y
\end{aligned}
$$

By expanding the $(y+x)^{m+n}$ term according to [63, eq. (1.111)] and substituting in (209) yields (211), as shown at the bottom of the page.

Importantly, the integral in (211) can be expressed in closed-form with the aid of [86, eq. (3.35.1.9)]. As a result (81) is deduced, which completes the proof.

$$
\begin{aligned}
I e_{m, n}(x ; a)= & \frac{(m+n) !_{2} F_{1}\left(\frac{m+n+1}{2}, \frac{m+n}{2}+1 ; 1+n ; \frac{1}{a^{2}}\right)}{2^{n} a^{m+n+1} n !} \\
& -\frac{e^{-x(1+a)}}{2^{n} n !} \sum_{l=0}^{m+n}\left(\begin{array}{c}
m+n \\
l
\end{array}\right) x^{m+n-l} \int_{0}^{\infty} y^{l} e^{-y(1+a)}{ }_{2} F_{1}\left(n+\frac{1}{2}, 1+2 n, 2(x+y)\right) \mathrm{d} y .
\end{aligned}
$$




\section{APPENDIX J}

\section{PROOF OF THEOREM 9}

By reversing the integral limits and applying [63, eq. (9.238.2)] it follows that,

$$
\begin{aligned}
I e_{-n, n}(x ; a)= & \int_{0}^{\infty} \frac{{ }_{1} F_{1}\left(n+\frac{1}{2}, 1+2 n, 2 y\right)}{2^{n} n ! e^{y(1+a)}} \mathrm{d} y \\
& -\int_{x}^{\infty} \frac{{ }_{1} F_{1}\left(n+\frac{1}{2}, 1+2 n, 2 y\right)}{2^{n} n ! e^{y(1+a)}} \mathrm{d} y .
\end{aligned}
$$

The first integral can be evaluated analytically with the aid of [63, eq. (7.521)]. To this effect and by setting in the second integral $u=2 y+x$ and carrying out some basic algebraic manipulations one obtains,

$$
\begin{aligned}
I e_{-n, n}(x ; a)= & \frac{{ }_{2} F_{1}\left(n+\frac{1}{2}, 1 ; 1+2 n ; \frac{2}{1+a}\right)}{2^{n} n !(1+a)} \\
& -\int_{0}^{\infty} \frac{{ }_{1} F_{1}\left(n+\frac{1}{2}, 1+2 n, u+2 x\right)}{2^{n+1} n ! e^{x(1+a)} e^{(1+a) \frac{u}{2}}} \mathrm{~d} u .
\end{aligned}
$$

The above integral can be expressed in terms of the Humbert function $\Phi_{1}$ according to [86, eq. (3.35.1.9)]. To this effect, one obtains the closed-form expression in (82).

For the special case that $m=n=0$ in (78) and setting $u=a y \Rightarrow y=u / a$ and $\mathrm{d} / \mathrm{d} t=a$ one obtains,

$$
I e_{0,0}(x ; a)=\int_{0}^{x} e^{-a y} I_{0}(y) \mathrm{d} y
$$

which can be equivalently expressed as,

$$
I e_{0,0}(x ; a)=\int_{0}^{a x} \frac{e^{-u}}{a} I_{0}\left(\frac{u}{a}\right) \mathrm{d} u .
$$

The above integral can be expressed in closed-form according to [62, eq. (2c)]. Thus, by substituting in (215) and after basic algebraic manipulations, eq. (83) is deduced. This completes the proof.

\section{APPENDIX K ProOf OF PROPOSITION 6}

By making the necessary variable transformation in [83, eq. (19)] and substituting in (89) one obtains,

$$
I e_{m, n}(x ; a) \simeq \sum_{l=0}^{L} \int_{0}^{x} \frac{\Gamma(L+l) L^{1-2 l} y^{m+n+2 l}}{l !(L-l) !(n+l) ! 2^{n+2 l} e^{a y}} \mathrm{~d} y .
$$

The above integral can be expressed in closed-form according to [63, eq. (3. 381.3)], namely,

$$
\int_{0}^{x} y^{m+n+2 l} e^{-a y} \mathrm{~d} y=\frac{\gamma(m+n+2 l+1, a x)}{a^{m+n+2 l+1}} .
$$

By substituting (217) into (216) equation (89) is deduced. To this effect and as $L \rightarrow \infty$, the terms

$$
\frac{\Gamma(L+l) L^{1-2 l}}{(L-l) !}
$$

vanish and (89) becomes the exact infinite series in (90), which completes the proof.

\section{ACKNOWLEDGMENTS}

The authors would like to thank the Associate Editor and the anonymous reviewers for their constructive comments.

\section{REFERENCES}

[1] J. I. Marcum, "A statistical theory of target detection by pulsed radar: Mathematical appendix," RAND Corp., Santa Monica, CA, USA, Tech. Rep., 1948.

[2] J. I. Marcum, "Table of $Q$-functions, U.S. air force project RAND res. memo. M-339, ASTIA document AD 116545," RAND Corp. Santa Monica, CA, USA, Tech. Rep., 1950.

[3] J. Marcum, "A statistical theory of target detection by pulsed radar," IRE Trans. Inf. Theory, vol. IT-6, no. 2, pp. 59-267, Apr. 1960.

[4] P. Swerling, "Probability of detection for fluctuating targets," IRE Trans. Inf. Theory, vol. IT-6, no. 2, pp. 269-308, Apr. 1960.

[5] J. G. Proakis, Digital Communications, 3rd ed. New York, NY, USA: McGraw-Hill, 1995.

[6] D. A. Shnidman, "The calculation of the probability of detection and the generalized Marcum $Q$-function," IEEE Trans. Inf. Theory, vol. 35, no. 2, pp. 389-400, Mar. 1989.

[7] C. W. Helstrom, "Computing the generalized Marcum $Q$-function," IEEE Trans. Inf. Theory, vol. 38, no. 4, pp. 1422-1428, Jul. 1992.

[8] M. K. Simon, "A new twist on the Marcum $Q$-function and its application," IEEE Commun. Lett., vol. 2, no. 2, pp. 39-41, Feb. 1998.

[9] A. H. Nuttall, "Some integrals involving the $Q$-function," Naval Underwater Syst. Center, New London Lab., New London, CT, USA, Tech. Rep. 4297, 1972.

[10] M. K. Simon and M.-S. Alouini, "Exponential-type bounds on the generalized Marcum $Q$-function with application to error probability analysis over fading channels," IEEE Trans. Commun., vol. 48, no. 3, pp. 359-366, Mar. 2000.

[11] M. K. Simon, "The Nuttall $Q$ function-its relation to the Marcum $Q$ function and its application in digital communication performance evaluation," IEEE Trans. Commun., vol. 50, no. 11, pp. 1712-1715, Nov. 2002.

[12] A. H. Heatley, "A short table of the Toronto functions," Trans. Roy. Soc. Canada, vol. 37, 1943.

[13] H. Sagon, "Numerical calculation of the incomplete Toronto function," Proc. IEEE, vol. 54, no. 8, p. 1095, Aug. 1966.

[14] R. A. Fisher, "The general sampling distribution of the multiple correlation coefficient," Proc. Roy. Soc. London, vol. 121, no. 788, pp. 654-673, Dec. 1928.

[15] S. O. Rice, "Statistical properties of a sine wave plus random noise," Bell Syst. Tech. J., vol. 27, pp. 109-157, Jan. 1948.

[16] J. H. Roberts, Angle Modulation: The Theory of System Assessment. Stevenage, U.K.: Peregrinus, 1977.

[17] R. F. Pawula, S. O. Rice, and J. H. Roberts, "Distribution of the phase angle between two vectors perturbed by Gaussian noise," IEEE Trans. Commun., vol. COM-30, no. 8, pp. 1828-1841, Aug. 1982.

[18] B. Tan, T. T. Tjhung, C. Teo, and P. Leong, "Series representations for Rice's Ie function," IEEE Trans. Commun., vol. COM-32, no. 12, pp. 1342-1344, Dec. 1984.

[19] M. M. Agrest, "Bessel function expansions of incomplete Lipschitz-Hankel integrals," USSR Comput. Math. Math. Phys., vol. 11, no. 5, pp. 40-54, May 1971.

[20] M. M. Agrest and M. Z. Maksimov, Theory of Incomplete Cylindrical Functions and Their Applications. New York, NY, USA: Springer-Verlag, 1971.

[21] A. Miller, "Incomplete Lipschitz-Hankel integrals of Bessel functions," J. Math. Anal. Appl., vol. 140, no. 2, pp. 476-484, 1989.

[22] S. L. Dvorak, "Applications for incomplete Lipschitz-Hankel integrals in electromagnetics," IEEE Antennas Propag. Mag., vol. 36, no. 6, pp. 26-32, Dec. 1994.

[23] M. K. Simon and M.-S. Alouini, Digital Communication Over Fading Channels, 2nd ed. New York, NY, USA: Wiley, 2005.

[24] P. Loskot and N. C. Beaulieu, "Prony and polynomial approximations for evaluation of the average probability of error over slow-fading channels," IEEE Trans. Veh. Technol., vol. 58, no. 3, pp. 1269-1280, Mar. 2009.

[25] X. Li and J. Cheng, "Asymptotic error rate analysis of selection combining on generalized correlated Nakagami- $m$ channels," IEEE Trans. Commun., vol. 60, no. 7, pp. 1765-1771, Jul. 2012. 
[26] S. P. Herath, N. Rajatheva, and C. Tellambura, "Energy detection of unknown signals in fading and diversity reception," IEEE Trans. Commun., vol. 59, no. 9, pp. 2443-2453, Sep. 2011.

[27] J. F. Paris, "Nakagami- $q$ (Hoyt) distribution function with applications," Electron. Lett., vol. 45, no. 4, pp. 210-211, Feb. 2009.

[28] J. F. Paris and D. Morales-Jiménez, "Outage probability analysis for Nakagami- $q$ (Hoyt) fading channels under Rayleigh interference," IEEE Trans. Wireless Commun., vol. 9, no. 4, pp. 1272-1276, Apr. 2010.

[29] D. Morales-Jiménez and J. F. Paris, "Outage probability analysis for $\eta$ - $\mu$ fading channels," IEEE Commun. Lett., vol. 14, no. 6, pp. 521-523, Jun. 2010

[30] J.-C. Shen, E. Alsusa, and D. K. C. So, "Performance bounds on cyclostationary feature detection over fading channels," in Proc. IEEE WCNC, Shanghai, China, Apr. 2013, pp. 2971-2975.

[31] G. Cui, A. De Maio, M. Piezzo, and A. Farina, "Sidelobe blanking with generalized Swerling-chi fluctuation models," IEEE Trans. Aerosp. Electron. Syst., vol. 49, no. 2, pp. 982-1005, Apr. 2013.

[32] G. Alfano, A. De Maio, and A. M. Tulino, "A theoretical framework for LMS MIMO communication systems performance analysis," IEEE Trans. Inf. Theory, vol. 56, no. 11, pp. 5614-5630, Nov. 2010.

[33] D. Morales-Jimenez, J. F. Paris, and A. Lozano, "Outage probability analysis for MRC in $\eta$ - $\mu$ fading channels with co-channel interference," IEEE Commun. Lett., vol. 16, no. 5, pp. 674-677, May 2012.

[34] J. P. Pena-Martin, J. M. Romero-Jerez, and C. Tellez-Labao, "Performance of TAS/MRC wireless systems under Hoyt fading channels," IEEE Trans. Wireless Commun., vol. 12, no. 7, pp. 3350-3359, Jul. 2013.

[35] F. J. Lopez-Martinez, D. Morales-Jimenez, E. Martos-Naya, and J. F. Paris, "On the bivariate Nakagami- $m$ cumulative distribution function: Closed-form expression and applications," IEEE Trans. Commun., vol. 61, no. 4, pp. 1404-1414, Apr. 2013.

[36] Y. Sun, A. Baricz, M. Zhao, X. Xu, and S. Zhou, "Approximate average bit error probability for DQPSK over fading channels," Electron. Lett., vol. 45, no. 23, pp. 1177-1179, Nov. 2009.

[37] I. Trigui, A. Laourine, S. Affes, and A. Stephenne, "Performance analysis of mobile radio systems over composite fading/shadowing channels with co-located interference," IEEE Trans. Wireless Commun., vol. 8, no. 7, pp. 3448-3453, Jul. 2009.

[38] G. E. Corazza and G. Ferrari, "New bounds for the Marcum $Q$-function," IEEE Trans. Inf. Theory, vol. 48, no. 11, pp. 3003-3008, Nov. 2002

[39] R. Li and P. Y. Kam, "Computing and bounding the generalized Marcum $Q$-function via a geometric approach," in Proc. IEEE ISIT, Seattle, WA, USA, Jul. 2006, pp. 1090-1094.

[40] P. Y. Kam and R. Li, "Generic exponential bounds and erfc-bounds on the Marcum $Q$-function via the geometric approach," in Proc. IEEE GLOBECOM, San Francisco, CA, USA, Nov./Dec. 2006, pp. 1-5.

[41] X. Zhao, D. Gong, and Y. Li, "Tight geometric bound for Marcum $Q$-function," Electron. Lett., vol. 44, no. 5, pp. 340-341, Feb. 2008.

[42] P. Y. Kam and R. Li, "Computing and bounding the first-order Marcum $Q$-function: A geometric approach," IEEE Trans. Commun., vol. 56, no. 7, pp. 1101-1110, Jul. 2008

[43] Y. Sun and S. Zhou, "Tight bounds of the generalized Marcum $Q$-function based on log-concavity," in Proc. IEEE GLOBECOM, New Orleans, LA, USA, Nov./Dec. 2008, pp. 1-5.

[44] Y. Sun and A. Baricz, "Inequalities for the generalized Marcum $Q$-function," Appl. Math. Comput., vol. 203, no. 1, pp. 134-141, 2008.

[45] Á. Baricz and Y. Sun, "New bounds for the generalized Marcum $Q$-function," IEEE Trans. Inf. Theory, vol. 55, no. 7, pp. 3091-3100, Jul. 2009.

[46] V. M. Kapinas, S. K. Mihos, and G. K. Karagiannidis, "On the monotonicity of the generalized Marcum and Nuttall $Q$-functions," IEEE Trans. Inf. Theory, vol. 55, no. 8, pp. 3701-3710, Aug. 2009.

[47] Á. Baricz, "Tight bounds for the generalized Marcum $Q$-function," J. Math. Anal. Appl., vol. 360, no. 1, pp. 265-277, Dec. 2009.

[48] G. T. F. de Abreu, "Jensen-Cotes upper and lower bounds on the Gaussian $Q$-function and related functions," IEEE Trans. Commun., vol. 57, no. 11, pp. 3328-3338, Nov. 2009.

[49] R. Li, P. Y. Kam, and H. Fu, "New representations and bounds for the generalized Marcum $Q$-function via a geometric approach, and an application," IEEE Trans. Commun., vol. 58, no. 1, pp. 157-169, Jan. 2010

[50] Y. Sun, Á. Baricz, and S. Zhou, "On the monotonicity, log-concavity, and tight bounds of the generalized Marcum and Nuttall $Q$-functions," IEEE Trans. Inf. Theory, vol. 56, no. 3, pp. 1166-1186, Mar. 2010.
[51] H. Fu and P.-Y. Kam, "Exponential-type bounds on the first-order Marcum $Q$-function," in Proc. IEEE GLOBECOM, Houston, TX, USA, Dec. 2011, pp. 1-5

[52] I.-S. Koh and S. P. Chang, "Uniform bounds of first-order Marcum $Q$-function," IET Commun., vol. 7, no. 13, pp. 1331-1337, Sep. 2013.

[53] P. Y. Kam and R. Li, "A new geometric view of the firstorder Marcum $Q$-function and some simple tight erfc-bounds," in Proc. IEEE 63rd VTC Spring, Melbourne, Australia, May 2006, pp. 2553-2557.

[54] N. Ding and H. Zhang, "A flexible method to approximate Marcum $Q$-function based on geometric way of thinking," in Proc. 3rd ISCCSP, Saint Julian's, Malta, Apr. 2008, pp. 1351-1356.

[55] S. András, Á. Baricz, and Y. Sun, "The generalized Marcum $Q$-function: An orthogonal polynomial approach," Acta Univ. Sapientiae, Math. vol. 3, no. 1, pp. 60-76, Jan. 2011

[56] M. Z. Bocus, C. P. Dettmann, and J. P. Coon, "An approximation of the first order Marcum $Q$-function with application to network connectivity analysis," IEEE Commun. Lett., vol. 17, no. 3, pp. 499-502, Mar. 2013.

[57] Y. A. Brychkov, "On some properties of the Marcum $Q$ function," Integral Transf. Special Funct., vol. 23, no. 3, pp. 177-182, Mar. 2012.

[58] V. M. Kapinas, "Optimization and performance evaluation of digital wireless communication systems with multiple transmit and receive antennas," Ph.D. dissertation, Aristotle Univ. Thessaloniki, Thessaloniki, Greece, 2014.

[59] D. Morales-Jimenez, F. J. Lopez-Martinez, E. Martos-Naya, J. F. Paris, and A. Lozano, "Connections between the generalized Marcum $Q$-function and a class of hypergeometric functions," IEEE Trans. Inf. Theory, vol. 60, no. 2, pp. 1077-1082, Feb. 2014.

[60] Q. Shi and Y. Karasawa, "An intuitive methodology for efficient evaluation of the Nuttall $Q$-function and performance analysis of energy detection in fading channels," IEEE Trans. Wireless Commun., vol. 1 no. 2, pp. 109-112, Apr. 2012.

[61] Y. A. Brychkov, "On some properties of the Nuttall function $Q_{\mu, \nu}(a, b), "$ Integral Transf. Special Funct., vol. 25, no. 1, pp. 34-43, Jan. 2014

[62] R. F. Pawula, "Relations between the Rice $I e$-function and the Marcum $Q$-function with applications to error rate calculations," Electron. Lett., vol. 31, no. 24, pp. 2078-2080, Nov. 1995.

[63] I. S. Gradshteyn and I. M. Ryzhik, Table of Integrals, Series, and Products, 7th ed. New York, NY, USA: Academic, 2007.

[64] J. F. Paris, E. Martos-Naya, U. Fernández-Plazaola, and J. LopezFernandez, "Analysis of adaptive MIMO transmit beamforming under channel prediction errors based on incomplete Lipschitz-Hankel integrals," IEEE Trans. Veh. Technol., vol. 58, no. 6, pp. 2815-2824, Jul. 2009

[65] P. C. Sofotasios, "On special functions and composite statistical distributions and their applications in digital communications over fading channels, Ph.D. dissertation, Dept. Electron. Elect. Eng., Univ. Leeds, England, U.K., 2010

[66] P. C. Sofotasios and S. Freear, "A novel representation for the Nuttall $Q$-function," in Proc. IEEE ICWITS, Honolulu, HI, USA Aug./Sep. 2010, pp. 1-4.

[67] P. C. Sofotasios and S. Freear, "Novel expressions for the Marcum and one dimensional $Q$-functions," in Proc. 7th ISWCS, York, U.K., Sep. 2010, pp. 736-740.

[68] P. C. Sofotasios and S. Freear, "Simple and accurate approximations for the two dimensional Gaussian $Q$-function," in Proc. IEEE 73rd VTC Spring, Budapest, Hungary, May 2011, pp. 1-4.

[69] P. C. Sofotasios and S. Freear, "New analytic results for the incomplete Toronto function and incomplete Lipschitz-Hankel integrals," in Proc. IEEE SBMO/IMOC, Natal, Brazil, Oct./Nov. 2011, pp. $1-5$.

[70] S. Harput, P. C. Sofotasios, and S. Freear, "A novel composite statistical model for ultrasound applications," in Proc. IEEE IUS, Orlando, FL, USA, Oct. 2011, pp. 1-4

[71] P. C. Sofotasios and S. Freear, "Upper and lower bounds for the Rice Ie-function," in Proc. IEEE ATNAC, Melbourne, Australia, Nov. 2011 pp. $1-4$.

[72] P. C. Sofotasios and S. Freear, "Analytic expressions for the Rice $I e$-function and the incomplete Lipschitz-Hankel integrals," in Proc. IEEE Annu. INDICON, Hyderabad, India, Dec. 2011, pp. 1-6. 
[73] P. C. Sofotasios, K. Ho-Van, T. D. Anh, and H. D. Quoc, "Analytic results for efficient computation of the Nuttall $-Q$ and incomplete Toronto functions," in Proc. IEEE Int. Conf. ATC, Ho Chi Minh City, Vietnam, Oct. 2013, pp. 420-425.

[74] P. C. Sofotasios, M. Valkama, T. A. Tsiftsis, Y. A. Brychkov, S. Freear, and G. K. Karagiannidis, in Proc. CROWNCOM, Oulu, Finland, Jun. 2014, pp. 260-265.

[75] M. Abramowitz and I. A. Stegun, Handbook of Mathematical Functions with Formulas, Graphs, and Mathematical Tables. New York, NY, USA: Dover, 1974.

[76] W. N. Bailey, Appell's Hypergeometric Functions of Two Variables (Generalised Hypergeometric). Cambridge, U.K.: Cambridge Univ. Press, 1935, pp. 73-83 and 99-101.

[77] V. L. Deshpande, "Expansion theorems for the Kampé de Fériet function," Indagationes Math., vol. 74, pp. 39-46, 1971.

[78] H. Exton, Handbook of Hypergeometric Integrals: Theory, Applications, Tables, Computer Programs (Mathematics and Its Applications). Chichester, U.K.: Ellis Horwood Ltd., 1978.

[79] H. M. Srivastava and P. W. Karlsson, Multiple Gaussian Hypergeometric Series (Mathematics and Its Applications). Chichester, U.K.: Ellis Horwood Ltd., 1985.

[80] E. T. Whittaker and G. N. Watson, A Course in Modern Analysis, 4th ed. Cambridge, U.K.: Cambridge Univ. Press, 1990.

[81] H. Exton, "Transformations of certain generalized Kampé de Fériet functions II," J. Appl. Math. Stochasti Anal., vol. 10, no. 3, pp. 297-304, 1997.

[82] A. P. Prudnikov, Y. A. Brychkov, and O. I. Marichev, Integrals and Series: Elementary Functions, vol. 1, 3rd ed. New York, NY, USA: Gordon and Breach, 1992.

[83] L.-L. Li, F. Li, and F. B. Gross, "A new polynomial approximation for $J_{v}$ Bessel functions," Appl. Math. Comput., vol. 183, no. 2, pp. 1220-1225, 2006.

[84] P. Humbert, "Sur les fonctions hypercylindriques," Comptes Rendus Hebdomadaires Séances l'Acad. Sci., Paris, vol. 171, pp. 490-492, 1920.

[85] Y. A. Brychkov and N. Saad, "Some formulas for the Appell function $F_{1}\left(a, b, b^{\prime} ; c ; w, z\right)$," Integral Transf. Special Funct., vol. 23, no. 11, pp. 793-802, Nov. 2012.

[86] A. P. Prudnikov, Y. A. Brychkov, and O. I. Marichev, Integrals and Series: Direct Laplace Transforms, vol. 4. New York, NY, USA: Gordon and Breach, 1992.

[87] G. Fraidenraich and M. D. Yacoub, "The $\alpha-\eta-\mu$ and $\alpha-\kappa-\mu$ fading distributions," in Proc. 9th IEEE ISSTA, Manaus, Brazil, Aug. 2006, pp. 16-20.

[88] R. Cogliatti and R. A. Amaral de Souza, "A near-100\% efficient algorithm for generating $\alpha-\kappa-\mu$ and $\alpha-\eta-\mu$ variates," in Proc. IEEE VTC-Fall, Las Vegas, NV, USA, Sep. 2013, pp. 1-5.

[89] M. D. Yacoub, "The $\alpha-\mu$ distribution: A physical fading model for the Stacy distribution," IEEE Trans. Veh. Technol., vol. 56, no. 1, pp. 27-34, Jan. 2007.

[90] M. D. Yacoub, "The $\kappa-\mu$ distribution and the $\eta-\mu$ distribution," IEEE Antennas Propag. Mag., vol. 49, no. 1, pp. 68-81, Feb. 2007.

[91] G. Fraidenraich and M. D. Yacoub, "The $\lambda-\mu$ general fading distribution," in Proc. SMBO/IEEE MTT-S IMOC, Foz do Iguaçu, Brazil, Sep. 2003, pp. 49-54.

[92] A. Goldsmith and P. P. Varaiya, "Capacity of fading channels with channel side information," IEEE Trans. Inf. Theory, vol. 43, no. 6 , pp. 1986-1992, Nov. 1997

[93] M.-S. Alouini and A. J. Goldsmith, "Capacity of Rayleigh fading channels under different adaptive transmission and diversity-combining techniques," IEEE Trans. Veh. Technol., vol. 48, no. 4, pp. 1165-1181, Jul. 1999.

[94] S. K. Jayaweera and H. V. Poor, "On the capacity of multiple-antenna systems in Rician fading," IEEE Trans. Wireless Commun., vol. 4, no. 3 , pp. 1102-1111, May 2005.

[95] A. Maaref and S. Aissa, "Capacity of MIMO Rician fading channels with transmitter and receiver channel state information," IEEE Trans. Wireless Commun., vol. 7, no. 5, pp. 1687-1698, May 2008.

[96] G. Alfano, A. Lozano, A. M. Tulino, and S. Verdú, "Mutual information and eigenvalue distribution of MIMO Ricean channels," in Proc. IEEE Int. Symp. Inf. Theory Appl. (ISITA), Parma, Italy, Oct. 2004, pp. 1-6.
Paschalis C. Sofotasios was born in Volos, Greece in 1978. He received the M.Eng. degree from the University of Newcastle upon Tyne, UK, in 2004, the M.Sc. degree from the University of Surrey, UK, in 2006, and the Ph.D. degree from the University of Leeds, UK, in 2011. His Master's studies were funded by a scholarship from UK-EPSRC and his Doctoral studies were sponsored by UK-EPSRC and Pace plc. He was a PostDoctoral Researcher at the University of Leeds until August 2013 and he has been a Visiting Researcher at the University of California, Los Angleles, USA, Aristotle University of Thessaloniki, Greece and Tampere University of Technology, Finland. Since Fall 2013, he is a Post- Doctoral Research Fellow at the Department of Electronic and Communications Engineering of Tampere University of Technology and at the Wireless Communications Systems Group of Aristotle University of Thessaloniki. His research interests are in the areas of fading channel characterisation, cognitive radio, cooperative communications, optical wireless communications as well as in the theory of special functions and statistical distributions. He received a 2012 Exemplary Reviewer award by IEEE COMMUNICATION LETTERS and the best paper award at the ICUFN' 13

Theodoros A. Tsiftsis (S'02-M'04-SM'10) was born in Lamia, Greece, in 1970. He received the B.Sc. degree in physics from the Aristotle University of Thessaloniki, Greece, in 1993; the M.Sc. degree in digital systems engineering from the Heriot-Watt University, Edinburgh, U.K., in 1995; the M.Sc. degree in decision sciences from the Athens University of Economics and Business, Greece, in 2000; and the Ph.D. degree in electrical engineering from the University of Patras, Greece, in 2006.

$\mathrm{He}$ worked as a Telecom Engineer in Philips Business Communications B.V., Philips Hellas S.A., Athens, Greece between 1997-2002. Currently, he is an Assistant Professor in the Department of Electrical Engineering at Technological Educational Institute of Central Greece, and also a collaborative researcher with the Wireless Communications Systems Group (WCSG), Department of Electrical \& Computer Engineering, Aristotle University of Thessalonki, Greece. He is the author of more than 80 technical papers in scientific journals and international conferences. His research interests include the broad areas of cooperative communications, communication theory, wireless communications, and optical wireless communication systems.

Dr. Tsiftsis acts as a regular reviewer for several international journals and he is member of the Editorial Boards of IEEE TRANSACTIONS ON COMMUNiCATIONS and IEEE COMMUNICATIONS LETTERS.

Yury A. Brychkov was born in Moscow, Russia, in 1944. He was educated at the Mathematical- Mechanical Faculty of the Lomonossov Moscow State University and received the Diploma in mathematics in 1966. He was a post-graduate student at the Department of Quantum Field Theory of the Steklov Mathematical Institute of the Russian Academy of Sciences between 1966-1969. He joined the Dorodnicyn Computing Center of the Russian Academy of Sciences in 1969 and he received his Ph.D. in 1971. He has been a Senior Researcher with the same Institute and during the period 2000-2002, he was a Visiting Researcher in the Symbolic Computation Group of the University of Waterloo (Canada). His current research interests are in special functions, integral transformations and computer algebra. $\mathrm{He}$ is an author and co-author of 9 handbooks in these fields, including the 5-volume set Integrals and Series (Gordon and Breach Science Publishers, 1986-1992).

Steven Freear (S'95-M'97-SM'11) gained his doctorate in 1997 and subsequently worked in the electronics industry for 7 years as a VLSI system designer. He was appointed Lecturer (Assistant Professor) and then Senior Lecturer (Associate Professor) at the School of Electronic and Electrical Engineering at the University of Leeds in 2006 and 2008, respectively. His main research interest is concerned with advanced analogue and digital signal processing for ultrasonic instrumentation and wireless communication systems. He teaches digital signal processing, microcontrollers/microprocessors, VLSI and embedded systems design, hardware description languages at both undergraduate and postgraduate level.

Dr. Freear is the Editor-in-Chief of the IEEE TRANSACTIONS ON UlTRASONICS, FERROELECTRICS AND FREQUENCY CONTROL. 
Mikko Valkama was born in Pirkkala, Finland, on November 27, 1975 He received the M.Sc .and Ph.D. Degrees (both with honours) in electrical engineering (EE) from Tampere University of Technology (TUT), Finland, in 2000 and 2001, respectively. In 2002, he received the Best Ph.D. Thesis award by the Finnish Academy of Science and Letters for his dissertation entitled "Advanced I/Q signal processing for wideband receivers: Models and algorithms." In 2003, he was working as a visiting researcher with the Communications Systems and Signal Processing Institute at SDSU, San Diego, CA. Currently, he is a Full Professor and Department Vice-Head at the Department of Electronics and Communications Engineering at TUT, Finland. $\mathrm{He}$ has been involved in organizing conferences, like the IEEE SPAWC'07 (Publications Chair) held in Helsinki, Finland. His general research interests include communications signal processing, estimation and detection techniques, signal processing algorithms for software defined flexible radios, cognitive radio, full-duplex radio, radio localization, 5G mobile cellular radio, digital transmission techniques such as different variants of multicarrier modulation methods and OFDM, and radio resource management for ad-hoc and mobile networks.
George K. Karagiannidis (M'96-SM'03-F'14) was born in Pithagorion, Samos Island, Greece. He received the University Diploma (5 years) and $\mathrm{Ph} . \mathrm{D}$ degree, in electrical and computer engineering from the University of Patras, in 1987 and 1999, respectively. From 2000 to 2004, he was a Senior Researcher at the Institute for Space Applications and Remote Sensing, National Observatory of Athens, Greece. In June 2004, he joined the faculty of Aristotle University of Thessaloniki, Greece where he is currently a Professor and Director of the Digital Telecommunications Systems and Networks Laboratory. In January 2014, he joined Khalifa University, UAE, as a Professor in the ECE Department and Coordinator of the ICT Cluster.

His research interests are in the broad area of digital communications systems with emphasis on communications theory, energy efficient MIMO and cooperative communications, cognitive radio, smart grid and optical wireless communications.

$\mathrm{He}$ is the author or co-author of more than 250 technical papers published in scientific journals and referred international conferences. He is also the author of the Greek edition of a book on Telecommunications Systems and co-author of the book Advanced Optical Wireless Communications Systems (Cambridge Publications, 2012). He is a co-recipient of the Best Paper Award of the Wireless Communications Symposium (WCS) in the IEEE International Conference on Communications (ICC07), Glasgow, U.K., June 2007.

Dr. Karagiannidis has been a member of Technical Program Committees for several IEEE conferences such as ICC, GLOBECOM, VTC, WCNC etc. In the past he was Editor for Fading Channels and Diversity of the IEEE TRANSACTIONS ON COMMUNICATIONS, Senior Editor of IEEE COMMUNICATIONS LETTERS and Editor of the EURASIP Journal of Wireless Communications and Networks. He was Lead Guest Editor of the special issue on Optical Wireless Communications of the IEEE Journal on SELECTED AREAS IN COMMUNICATIONS and Guest Editor of the special issue on Large-scale multiple antenna wireless systems.

Since January 2012 he is the Editor-in Chief of the IEEE COMMUNICATIONS LETTERS. 